\title{
Modeling of the Human Shoulder as a Parallel Mechanism Without Constraints
}

\author{
David Ingram $^{\mathrm{a}}$, Christoph Engelhardt ${ }^{\mathrm{b}}$, Alain Farron ${ }^{\mathrm{c}}$, Alexandre Terrier ${ }^{\mathrm{b}}$, Philippe Müllhaupt ${ }^{1 \mathrm{a}}$ \\ ${ }^{a}$ Laboratoire d'Automatique, Ecole Polytechnique Fédérale de Lausanne, Switzerland \\ ${ }^{b}$ Laboratory of Biomechanical Orthopedics, Ecole Polytechnique Fédérale de Lausanne, Switzerland \\ ${ }^{c}$ University Hospital Centre and University of Lausanne, Switzerland
}

\begin{abstract}
The synthesis of shoulder kinematics, either for simulation in a model or imitation in a robot, is a challenging task because of the contact between shoulder blade and ribcage. As the shoulder moves, the shoulder blade glides over the ribcage. In kinematic models used to predict musculoskeletal kinetics, the contact is included using equality constraints, creating interdependencies between the kinematic coordinates. Such interdependencies make motion planning complex. Robotic mechanisms often imitate the shoulder's end-effector kinematics but not the gliding shoulder blade architecture It is only recently that a gliding shoulder blade architecture has been mechanically achieved. The goal of this paper is to propose a novel kinematic parallel model of the shoulder that includes the contact without using constraints. Mechanically, the gliding architecture is replaced with a parallel architecture. A shoulder model with constraints is used to build the parallel model. It is shown that replacing the contact constraints with kinematically equivalent kinematic chains, leads to a 2-3 parallel platform model of the shoulder. The scaffold model and parallel model parameterisations of the shoulder's kinematics are analysed in terms of the forward kinematic map. The coordinate spaces of the kinematic maps are analysed, resulting in three minimal parameterisations. Each minimal parameterisation uses a set of coordinates equal to the number of degrees of freedom. The minimal coordinates are independent and considerably simplify motion planning.
\end{abstract}

Keywords: Human shoulder, scapulothoracic contact, parallel mechanisms, kinematics, coordinate reduction

\section{Introduction}

The shoulder's role in our daily interactions with our environment is essential and dysfunctions can be severely debilitating. As such, a considerable amount of research has been done on the shoulder, including musculoskeletal modelling for simulation purposes in clinical contexts $[1,2,3]$. The human shoulder is also a highly flexible system capable of supporting heavy loads and has a wide range of movements. Given these attractive characteristics, it has also been studied for the purposes of imitation in humanoid robots $[4,5,6]$. However, the shoulder remains a challenging system for a number of reasons, such as its kinematic complexity.

The shoulder can be considered as a parallel mechanism [7, 8]. The motion of the shoulder girdle with respect to the thorax results from the combined movement of three bones: The clavicle (collar bone), scapula (shoulder blade) and humerus (upper arm bone). The bones are serially connected together through three articulations, yielding a kinematically redundant system. Multiple configurations of the bones yield the same configuration of the elbow joint. This characteristic is what gives the upper limb such a vast workspace. The shoulder is a parallel mechanism because there is a contact between the scapula and thorax, called the scapulothoracic contact. It is not an articulation but is important kinematically as it guides the movement of the scapula relative to the thorax, reducing the number of degrees of freedom of the shoulder.

Unlike the other three articulations, the scapulothoracic contact cannot be characterised by a single kinematic pair

\footnotetext{
${ }^{1}$ Corresponding author at: EPFL-STI-IGM Station 9, CH-1015 Lausanne, Switzerland. Tel. +41 2169 33838. Fax. +41 21 693 2574. E-mail: philippe.muellhaupt@epfl.ch
}

Preprint submitted to Elsevier

January 5, 2016 
and is challenging from a modelling perspective $[6,9]$. Many musculoskeletal models parameterise the kinematics using joint angles and enforce the scapulothoracic contact with equality constraints $[7,1,10,11]$. The presence of constraints in the kinematic model limit the options for motion planning which is a crucial step in predicting musculoskeletal kinetics, one of the main purposes of musculoskeletal shoulder models. Solutions to the motion planning procedure are data-driven optimization $[12,13]$ or regression models of the clavicle and scapula's kinematics in terms of humeral kinematics $[14,15,16]$. Optimisation procedures require measuring kinematics on the real system which is a challenging task $[17,18]$ and regression models do not respect the constraints.

The scapulothoracic contact is also challenging from a robotics perspective. Given the shoulder's large workspace and load bearing capabilities, a number of robotic systems have been developed, using serial [19] and parallel [4] architectures to try and reproduce the shoulder's kinematics. However, it is only recently that robots have been developed, incorporating the scapulothoracic contact $[20,21]$. Indeed, the technology to build such robots, like smaller motors and efficient pneumatic artificial muscles, has only recently been developed [22]. In addition to the complexity of the scapulo-thoracic contact, there is a coordination between the movement of the scapula and humerus [23], called the scapulohumeral rhythm that is not easily reproduced. Thus, the shoulder's kinematics still present a serious challenge of synthesis for simulation purposes and reverse engineering.

The goal of this paper is to present a model of the shoulder that incorporates the parallel architecture without explicitly using constraints. The presentation is based on a detailed kinematic analysis of a model of the human shoulder, combining mathematical rigour and anatomical accuracy. The proposed model is built from a kinematic model of the shoulder parameterised by joint angles [24], considering the bones to be rigid bodies, the articulations to be ideal mechanical joints and represents the contact between scapula and thorax using two holonomic constraints. The model has seven degrees of freedom: nine kinematic coordinates subject to two equality constraints and is referred to as the natural kinematic model. The proposed model is constructed by replacing the constraints with kinematic chains, leading to an equivalent parallel mechanism description of the shoulder. These chains are parameterised with additional coordinates providing three alternative forward kinematic maps. The natural kinematic map and two others. The coordinate space topologies of all three maps are analysed, resulting in the definition of three different minimal parameterisations of the model's kinematics, each using seven independent coordinates. The paper concludes with a short discussion on the limitations of the model and the possible applications of the kinematic analysis.

\section{A Kinematic Shoulder Model}

\subsection{Geometric Model}

This section presents the kinematic shoulder model from which the parallel model is constructed. The model is similar to other models from the literature in its definition of reference frames and coordinates $[7,1]$. The present model parameterises bone movement relative to the thorax which is fixed (See [25] for anatomical references. Fig. 1). Each bone is represented by three points. The thorax is presented by four points, yielding a total of 13 points, defined in the following list:

1. IJ : Jugular Inscision, top of the sternum (thorax),

2. PX : Xyphoid Process, bottom of the sternum (thorax),

3. T8 : Eighth thoracic vertebrae (thorax),

4. C7 : Seventh cervical vertebrae (thorax),

5. CN : Conoid ligament attachement point (clavicle),

6. SC : Sternoclavicular articulation centre (clavicle),

7. $\mathrm{AC}:$ Acromioclavicular articulation centre (clavicle),
8. AA : Angulus acromialis (scapula),

9. TS : Trigonum Spinae (scapula),

10. AI : Angulus Inferior (scapula),

11. GH : Glenohumeral articulation centre (humerus),

12. EL : Lateral epicondyle of the humerus (humerus),

13. EM : Medial epicondyle (humerus).

Bone orientations are defined using four reference frames. A subindex is attributed to each reference frame ${ }^{2}$ $\left(\mathbf{i}_{i}, \mathbf{j}_{i}, \mathbf{k}_{i}, i=0,1,2,3\right)$ (Fig. 1). The thorax is defined as the carrier body and is attributed the subindex 0.

\footnotetext{
${ }^{2}$ Notation: Geometric points are defined by plane upper case lettres. Their vector position with respect to the centre of a reference frame is defined by a plane lower case letter with an arrow and the subindex associated with the reference frame. A matrix is defined by a bold upper case letter. Plane lower case letters with subindexes $x, y$ or $z$ define a vector's coordinates. The three bold lower case lettres $\mathbf{i}_{i}, \mathbf{j}_{i}, \mathbf{k}_{i}$ are the unit vectors of a reference frame.
} 
- Thorax Reference System $\mathcal{R}_{0}\left(\mathbf{i}_{0}, \mathbf{j}_{0}, \mathbf{k}_{0}\right)$ :

- Centre: $I J \equiv O_{0}$,

- $x$-axis $\left(\mathbf{i}_{0}\right)$ : normal to the plane defined by $I J, C 7, \frac{1}{2}(T 8+P X)-I J$, pointing to the right,

- $y$-axis $\left(\mathbf{j}_{0}\right)$ : normal to the $x$ and $z$ axes, pointing forwards,

- $z$-axis $\left(\mathbf{k}_{0}\right)$ : parallel to the line between the points $P_{1}=\frac{1}{2}(P X+C 7)$ and $P_{2}=\frac{1}{2}(T 8+I J)$.

- Clavicle Reference System $\mathcal{R}_{1}\left(\mathbf{i}_{1}, \mathbf{j}_{1}, \mathbf{k}_{1}\right)$ :

- Centre: $S C \equiv O_{1}$,

- $x$-axis ( $\mathbf{i}_{1}$ ): parallel to the line defined by $S C$ and $A C$, pointing to the right,

- $y$-axis $\left(\mathbf{j}_{1}\right)$ : normal to the plane defined by $\mathbf{i}_{1}$ and $\mathbf{k}_{0}$, pointing forwards,

- $z$-axis $\left(\mathbf{k}_{1}\right)$ : normal to the $x$ and $y$ axes, pointing upwards.

- Scapula Reference System $\mathcal{R}_{2}\left(\mathbf{i}_{2}, \mathbf{j}_{2}, \mathbf{k}_{2}\right)$ :

- Centre: $A C \equiv O_{2}$,

- $x$-axis $\left(\mathbf{i}_{2}\right)$ : parallel to the lined defined by $T S$ and $A A$, pointing to the right.

- $y$-axis $\left(\mathbf{j}_{2}\right)$ : normal to the plane defined by $\mathbf{i}_{2}$ and $A I-T S$ axes, pointing forwards,

- $z$-axis $\left(\mathbf{k}_{2}\right)$ : normal to the $x$ and $y$ axes, pointing upwards.

- Humerus Reference System $\mathcal{R}_{3}\left(\mathbf{i}_{3}, \mathbf{j}_{3}, \mathbf{k}_{3}\right)$ :

- Centre: $G H \equiv O_{3}$,

- $x$-axis $\left(\mathbf{i}_{3}\right)$ : normal to the $y$ and $z$ axes, pointing to the right,

- $y$-axis $\left(\mathbf{j}_{3}\right)$ : normal to the plane defined by $G H, E L$ and $E M$, pointing forwards,

- $z$-axis $\left(\mathbf{k}_{3}\right)$ : parallel to the line between the points $P_{1}=\frac{1}{2}(E L+E M)$ and $P_{2}=G H$.

\subsection{Joint Angle Kinematic Parameterisation}

The three articulations are modelled as ideal ball and socket joints, parameterised by a set of three angular coordinates [25].

$$
\vec{\Upsilon}_{i}=\left(\begin{array}{lll}
\psi_{i} & \vartheta_{i} & \varphi_{i}
\end{array}\right)^{T}, \quad i=1,2,3
$$

The three angular coordinates of $\vec{\Upsilon}_{i}$ are Euler angles and are all equal to zero when the reference frame $\mathcal{R}_{i}$ is aligned with the thorax or inertial frame $\mathcal{R}_{0}$.

The coordinate transformations between local bone frames $\mathcal{R}_{i}$ and the thorax reference frame $\mathcal{R}_{0}$, are defined by:

$$
\begin{aligned}
P_{0,1}: & \vec{p}_{0,1}=\vec{d}_{0,1}+\mathbf{R}_{1,0} \vec{p}_{1}, \\
P_{0,2}: & \vec{p}_{0,2}=\vec{d}_{0,2}+\mathbf{R}_{2,0} \vec{x}_{2}=\vec{d}_{0,1}+\mathbf{R}_{1,0} \vec{z}_{1}+\mathbf{R}_{2,0} \vec{p}_{2}, \\
P_{0,3}: & \vec{p}_{0,3}=\vec{d}_{0,3}+\mathbf{R}_{3,0} \vec{x}_{3}=\vec{d}_{0,1}+\mathbf{R}_{1,0} \vec{z}_{1}+\mathbf{R}_{2,0} \vec{z}_{2}+\mathbf{R}_{3,0} \vec{p}_{3} .
\end{aligned}
$$

The rotation matrices are defined by:

$$
\mathbf{R}_{i, 0}=\mathbf{R}_{z, i} \mathbf{R}_{y, i}^{T} \mathbf{R}_{x, i}, \quad \mathbf{R}_{x, i}=\left(\begin{array}{ccc}
1 & 0 & 0 \\
0 & \cos \left(\psi_{i}\right) & -\sin \left(\psi_{i}\right) \\
0 & \sin \left(\psi_{i}\right) & \cos \left(\psi_{i}\right)
\end{array}\right), \quad \mathbf{R}_{y, i}=\left(\begin{array}{rrr}
\cos \left(\vartheta_{i}\right) & 0 & -\sin \left(\vartheta_{i}\right) \\
0 & 1 & 0 \\
\sin \left(\vartheta_{i}\right) & 0 & \cos \left(\vartheta_{i}\right)
\end{array}\right), \quad \mathbf{R}_{z, i}=\left(\begin{array}{rrr}
\cos \left(\varphi_{i}\right) & -\sin \left(\varphi_{i}\right) & 0 \\
\sin \left(\varphi_{i}\right) & \cos \left(\varphi_{i}\right) & 0 \\
0 & 0 & 1
\end{array}\right)
$$

The vectors $\vec{d}_{0, i}, \vec{z}_{1}, \vec{z}_{2}$ and $\vec{w}_{2}$ parameterise the following geometric elements of the model.

- $\vec{d}_{0, i} \quad$ Vector from $I J$ to centre of frame $\mathcal{R}_{i}$ in $\mathcal{R}_{0}$.

- $\vec{z}_{1} \quad$ Vector from $S C$ to $A C$ in $\mathcal{R}_{1}$.

- $\vec{z}_{2} \quad$ Vector from $A C$ to $G H$ in $\mathcal{R}_{2}$. 


\subsection{Scapulothoracic Contact Model}

The scapulothoracic contact is the contact between the scapula and ribcage. It is modelled using an ellipsoid model $[1,26]$. The points $T S$ and $A I$ are constrained to remain on the surface of two separate ellipsoids (Fig. 1 (b)). Both ellipsoids have the same centre but different axial dimensions and are aligned with the inertial frame $\mathcal{R}_{0}$. They are constructed by fitting a single ellipsoid to the surface of the ribcage. This ellipsoid is then uniformly dilated twice (a constant is added to all three axial dimensions). The dilation constants are defined such that the points $T S$ and $A I$ lie on the surface of their respective ellipsoids. This yields two holonomic scleronomic constraints of the form:

$$
\begin{array}{ll}
\Phi_{T S}\left(\vec{\Upsilon}_{1}, \vec{\Upsilon}_{2}\right)=\left(\vec{u}_{0,2}-\vec{e}_{0}\right)^{T} \mathbf{E}_{T S}\left(\vec{u}_{0,2}-\vec{e}_{0}\right)-1=0, & T S_{0,2}: \vec{u}_{0,2}=\vec{d}_{0,2}+\mathbf{R}_{2,0} \vec{u}_{2}, \\
\Phi_{A I}\left(\vec{\Upsilon}_{1}, \vec{\Upsilon}_{2}\right)=\left(\vec{v}_{0,2}-\vec{e}_{0}\right)^{T} \mathbf{E}_{A I}\left(\vec{v}_{0,2}-\vec{e}_{0}\right)-1=0, & A I_{0,2}: \vec{v}_{0,2}=\vec{d}_{0,2}+\mathbf{R}_{2,0} \vec{v}_{2},
\end{array}
$$

where $\vec{u}_{0,2}$ and $\vec{v}_{0,2}$ are the vectors from the thorax origin to the points $T S$ and $A I$, in the thorax reference frame.The matrices $\mathbf{E}_{T S}$ and $\mathbf{E}_{A I}$ are the quadric matrices associated to each ellipsoid and $\vec{e}_{0}$ is the ellipsoid's common centre in the inertial frame $\mathcal{R}_{0}$.

$$
\mathbf{E}_{k}=\left(\begin{array}{ccc}
\frac{1}{a_{k}^{2}} & 0 & 0 \\
0 & \frac{1}{b_{k}^{2}} & 0 \\
0 & 0 & \frac{1}{c_{k}^{2}}
\end{array}\right), \quad \begin{aligned}
& a_{k}=a+\mu_{k}, \quad b_{k}=b+\mu_{k}, \quad c_{k}=c+\mu_{k}, \\
& k=T S, A I, a, b, c \text { : ellipsoid half-axis dimensions. }
\end{aligned}
$$

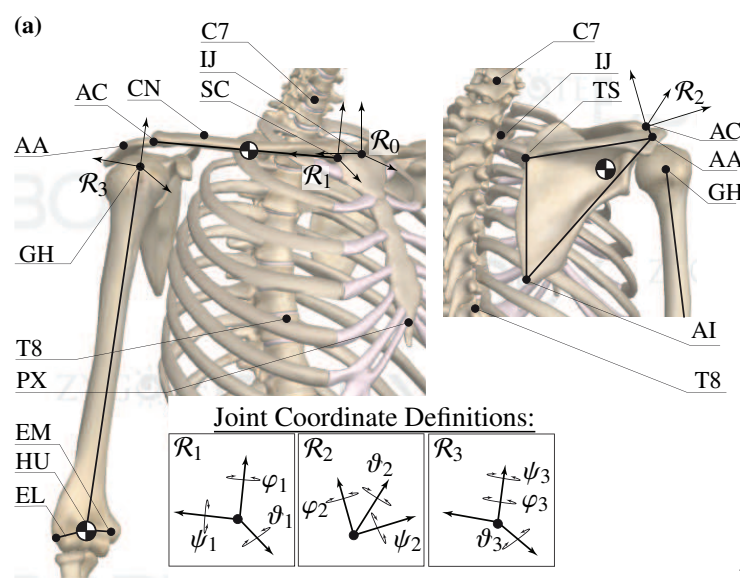

(b)

(c)

TS Ellipsoid Gliding Surface
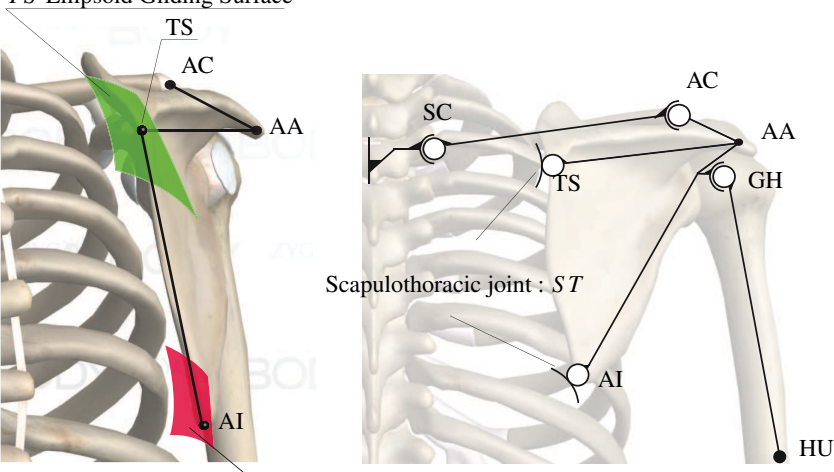

Figure 1: Illustration of (a) the bony landmarks, reference frames and joint coordinates. (b) the scapulothoracic contact model and (c) the kinematic shoulder model.

\subsection{Forward Kinematic Map}

The model's forward kinematics map the nine joint coordinates $\vec{q}=\left(\vec{\Upsilon}_{1}^{T}, \vec{\Upsilon}_{2}^{T}, \vec{\Upsilon}_{3}^{T}\right)^{T}$ to the configuration (position and orientation) of the humerus. The position is defined as the humeroulnar joint centre $H U_{0,3}$. The map is subject to the scapulothoracic contact constraints.

$$
\begin{aligned}
\Xi_{S}: \mathscr{Q}_{S} & \rightarrow \mathscr{W}_{S}, \\
& \vec{q} \mapsto \Xi_{S}(\vec{q})=C_{e}=\left(\vec{\epsilon}_{0,3}^{T}, \vec{\Upsilon}_{3}^{T}\right)^{T}, \quad H U_{0,3}: \vec{\epsilon}_{0,3}=\vec{d}_{0,3}+\mathbf{R}_{3,0} \vec{\epsilon}_{3}, \\
\text { s.t. } \quad & \Phi_{T S}\left(\vec{\Upsilon}_{1}, \vec{\Upsilon}_{2}\right)=\left(\vec{u}_{0,2}-\vec{e}_{0}\right)^{T} \mathbf{E}_{T S}\left(\vec{u}_{0,2}-\vec{e}_{0}\right)-1=0, \\
& \Phi_{A I}\left(\vec{\Upsilon}_{1}, \vec{\Upsilon}_{2}\right)=\left(\vec{v}_{0,2}-\vec{e}_{0}\right)^{T} \mathbf{E}_{A I}\left(\vec{v}_{0,2}-\vec{e}_{0}\right)-1=0 .
\end{aligned}
$$

The vector position of the end-effector $\vec{\epsilon}_{0,3}$ is defined according to the transformations (4). The configuration space $\mathscr{Q}_{S}$ is a subset of $S O(3) \times S O(3) \times S O(3)$ [27] cf. appendix Appendix A for details on $S O(3)$ and $\left.S E(3)\right)$. The work 
space $\mathscr{W}_{S}$ is a subset of $S E(3)=\mathbb{R}^{3} \ltimes S O(3)$. This parameterisation of the model will be referred to as the natural kinematic map because it uses joint coordinates.

The model is kinematically redundant [28]. Multiple configurations of the bones yield the same configuration of the end-effector, defined as the distal end of the humerus. The configuration of the humerus is defined by six degrees of freedom. There are nine kinematic coordinates, parameterising the model's configuration, subject to two holonomic scleronomic constraints. Using the Chebychev-Grübler-Kutzbach formula, the kinematic shoulder model has seven degrees of freedom [29].

$$
M=6 n-\sum_{j=1}^{m}\left(6-k_{j}\right)=7,
$$

where $n=3$ is the number of links, $m=5$ is the number of kinematic joints and $k_{j}$ is their associated degrees of freedom. There are three spherical kinematic pairs with three degrees of freedom each and there are two constraints, defining two spherical slider kinematic pairs with five degrees of freedom each. The model is redundant of degree one [30].

\section{A Parallel Platform Kinematic Shoulder Model}

The previous section presented a kinematic model of the shoulder where the scapulothoracic contact is modelled using two equality constraints. This section presents how these constraints are replaced with kinematic chains, yielding a parallel platform model of the shoulder. The presentation begins by showing that a Euclidean displacement, describing the configuration (position and orientation) of a body, has a mechanical description, i.e. there is a kinematic chain between a fixed point and the body. The same construction is then applied to a constrained rigid body and finally extended to the shoulder model previously presented.

All rigid body motions occur in Euclidean space and are described by direct isometries in the special Euclidean group is $S E(3)=\mathbb{R}^{3} \times S O(3)$. The configuration $C_{0, i}$ of a three dimensional rigid body $\mathscr{B}_{i}$ in $S E(3)$, is defined by a Euclidean displacement with respect to the inertial reference frame. Any point $P_{0, i}$ on the body is defined in the inertial frame by:

$$
P_{0, i}: \vec{p}_{0, i}=\vec{d}_{0, i}+\mathbf{R}_{i, 0} \vec{p}_{i}
$$

If the body is free, the displacement is parameterised by six independent coordinates. The three cartesian coordinates of the centre of the body's frame with respect to the inertial frame: $\vec{d}_{0, i}$. The three rotation angles of the body frame about the inertial frame: $\mathbf{R}_{i, 0}(\psi, \vartheta, \varphi)$. The three cartesian coordinates parameterise three, serially connected, orthogonal prismatic pairs used in mechanisms with linear guides. The combination of three orthogonal linear guides is the mechanical implementation of the cartesian coordinate chart on $\mathbb{R}^{3}$. The three rotation angles parameterise the spherical kinematic pair used in mechanisms with ball and socket joints. The ball and socket joint is the mechanical implementation of the Euler angle chart on $S O(3)$. Thus, a free-moving rigid body can be connected to a fixed point by a kinematic chain. The chain is three serially connected prismatic pairs, followed by a spherical pair. Its workspace is $S E(3)$ and it is the mechanical implementation of $S E(3)$.

An alternate mechanism is obtained using spherical coordinates $(\rho, \alpha, \beta)$ to chart $\mathbb{R}^{3}$. The cartesian coordinates of the displacement (12) are replaced by spherical coordinates.

$$
\vec{d}_{0, i}=\left(\begin{array}{c}
x_{0, i} \\
y_{0, i} \\
z_{0, i}
\end{array}\right)=\left(\begin{array}{c}
\rho_{d} \cos \left(\alpha_{d}\right) \sin \left(\beta_{d}\right) \\
\rho_{d} \sin \left(\alpha_{d}\right) \sin \left(\beta_{d}\right) \\
\rho_{d} \cos \left(\beta_{d}\right)
\end{array}\right) .
$$

The mechanical implementation of the spherical coordinate chart on $\mathbb{R}^{3}$ is defined by the following theorem.

Theorem 1 (Mechanical Description of $\mathbb{R}^{3}$ ). Any point in the three-dimensional Euclidean space $\mathbb{R}^{3}$ can be reached by a mechanism using a linear prismatic pair connected to a fixed point through a universal pair. 
Proof. Any point $P$ in $\mathbb{R}^{3}$ is described with respect to a fixed point $O$ in a fixed frame by three cartesian coordinates, defining the identity chart on $\mathbb{R}^{3}$. Each point is identified with itself and this atlas defines the standard smooth structure of $\mathbb{R}^{3}$. Another possible parameterisation is spherical coordinates $(\rho, \alpha, \beta)$, defining the following chart on $\mathbb{R}^{3}$ :

$$
\begin{aligned}
\phi: \mathbb{R}_{+} \times[0,2 \pi[\times[0, \pi] & \rightarrow \mathscr{U} \subset \mathbb{R}^{3}, \\
(\rho, \alpha, \beta) & \mapsto \phi(\rho, \alpha, \beta)=\vec{p}=\left(\begin{array}{c}
x \\
y \\
z
\end{array}\right)=\left(\begin{array}{c}
\rho \cos (\alpha) \sin (\beta) \\
\rho \sin (\alpha) \sin (\beta) \\
\rho \cos (\beta)
\end{array}\right) .
\end{aligned}
$$

The inverse chart is defined by:

$$
\begin{aligned}
\phi^{-1}: \mathscr{U} \subset \mathbb{R}^{3} \rightarrow \mathbb{R}_{+} \times[0,2 \pi[\times[0, \pi] \\
(x, y, z) \mapsto \phi^{-1}(x, y, z)=\left(\begin{array}{c}
\rho \\
\alpha \\
\beta
\end{array}\right)=\left(\begin{array}{c}
\sqrt{x^{2}+y^{2}+z^{2}} \\
\arctan \left(\frac{y}{x}\right) \\
\arctan \left(\frac{\sqrt{x^{2}+y^{2}}}{z}\right)
\end{array}\right) .
\end{aligned}
$$

These charts are local smooth charts. A single set of spherical coordinates does not uniquely cover $\mathbb{R}^{3}$. For a fixed value of $\rho \in \mathbb{R}_{+}, \alpha$ and $\beta$ chart $S^{2}$, requiring at least two charts. For instance, the north and south pole stereographic projections. Thus, $\mathbb{R}^{3}$ is locally equivalent to $\mathbb{R}_{+} \times S^{2}$. Spherical coordinates do chart $\mathbb{R}^{3}$ and the chart (14) can be written in the following form:

$$
P_{0}: \vec{p}_{0, i}=\left(\begin{array}{l}
x_{0, i} \\
y_{0, i} \\
z_{0, i}
\end{array}\right)=\left(\begin{array}{ccc}
\cos (\alpha) & -\sin (\alpha) & 0 \\
\sin (\alpha) & \cos (\beta) & 0 \\
0 & 0 & 1
\end{array}\right)\left(\begin{array}{ccc}
\cos (\beta) & 0 & \sin (\beta) \\
0 & 1 & 0 \\
\sin (\beta) & 0 & \cos (\beta)
\end{array}\right)\left(\begin{array}{l}
0 \\
0 \\
\rho
\end{array}\right) .
$$

The vector $\left(\begin{array}{lll}0 & 0 & \rho\end{array}\right)^{T}$, with $\rho \in \mathbb{R}_{+}$, parameterises an infinite linear prismatic kinematic pair. There is one coordinate parameterising a one-dimensional axes $[31,32,33]$. The two successive rotations around intersecting axes parameterise an ideal universal pair $[33,34,35]$. Thus, the spherical coordinate parameterisation of $\mathbb{R}^{3}$ can be implemented mechanically. The mechanism uses an infinite linear guide connected to a fixed point through a universal joint.

The special Euclidean group $S E(3)$ is charted by three spherical coordinates and three Euler angles. Such a chart has a mechanical implementation. The mechanism has a universal joint fixed to a point in the inertial frame. An infinite linear guide is attached, at one end, to the universal joint and allowed to freely rotate around the fixed point. At the other end, there is a ball and socket joint connecting the body to the prismatic joint (Fig: 2).
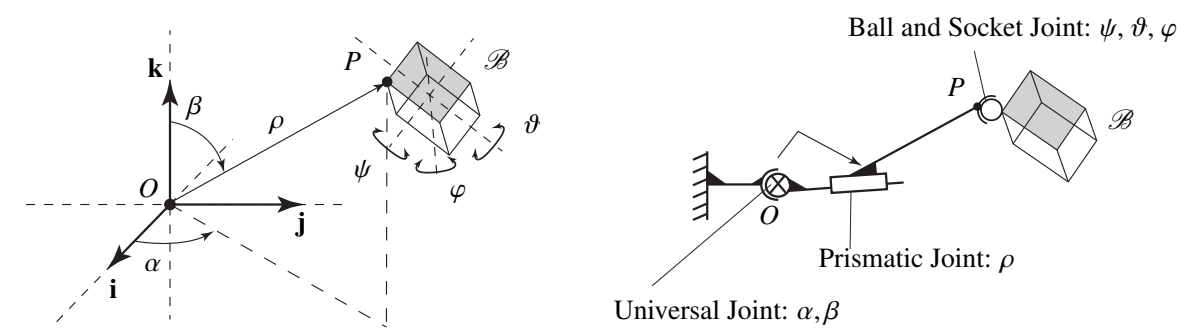

Figure 2: Illustration of the a mechanism with an end-effector workspace that is SE(3), as described in Theorem 1, section 3.

A rigid body, attached to two-dimensional surface in $\mathbb{R}^{3}$ through a single point is a body with five degrees of freedom. Two translational degrees of freedom of the point gliding over the surface and three rotational degrees of freedom. This situation can be mechanically implemented using the mechanism previously defined to implement $S E(3)$. Since the body can rotate around the contact point with three degrees of freedom. The ball and socket joint remains independent in the mechanism and it is one of the spherical coordinates that becomes dependent. 
The point $P$ on the body is now constrained to glide on a two-dimensional algebraic surface $\mathcal{S}$ defined by a smooth function of the coordinates of $P$.

$$
\mathcal{S}: f(x, y, z)=f(\rho, \alpha, \beta)=0 .
$$

Constraining $P$ to remain on an algebraic surface $\mathcal{S}$ is, to enforce a dependency between the coordinates. One of them is function of the others. For instance, $\rho=\rho\left(\alpha_{s}, \beta_{s}\right)$. The subindex $s$ indicates that $\left(\alpha_{s}, \beta_{s}\right)$ parameterise the surface. The three angles $(\psi, \vartheta, \varphi)$ remain independent. Mechanically, the prismatic joint's length is now function of both of the universal joint's angles. The prismatic joint is said to be passive, and cannot be actuated independently from the motion of the universal joint (Fig. 3).
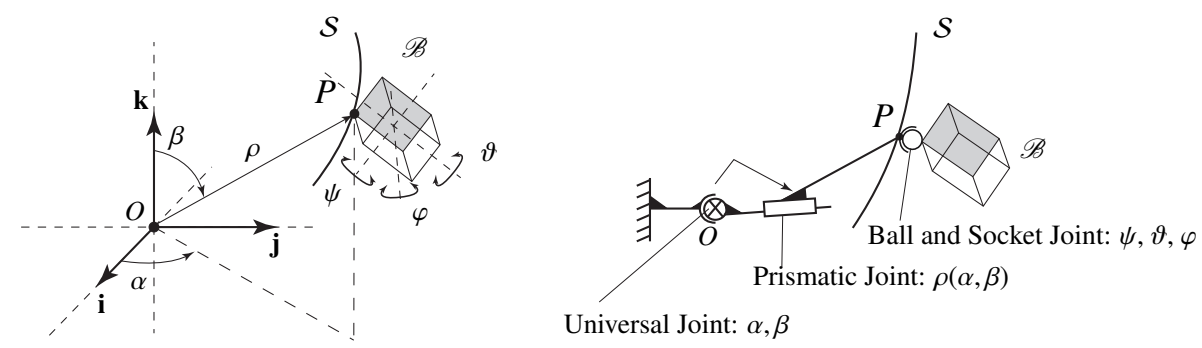

Figure 3: Illustration of the mechanical equivalent of a rigid body's motion on a two-dimensional, algebraic surface as described in section 3.

In the shoulder model, the scapula is a rigid body with two points that are in contact with an ellipsoid which is a two-dimensional surface in $\mathbb{R}^{3}$. The particular case of $\mathcal{S}$ being an ellipsoid imposes the following relation on the spherical coordinates $(\alpha, \beta, \rho)$ :

$$
\left(\begin{array}{c}
\rho \cos (\alpha) \sin (\beta) \\
\rho \sin (\alpha) \sin (\beta) \\
\rho \cos (\beta)
\end{array}\right)=\left(\begin{array}{c}
a_{e} \cos \left(\alpha_{e}\right) \sin \left(\beta_{e}\right) \\
b_{e} \sin \left(\alpha_{e}\right) \sin \left(\beta_{e}\right) \\
c_{e} \cos \left(\beta_{e}\right)
\end{array}\right), \quad \begin{aligned}
& (\alpha, \beta): \text { Universal joint coordinates } \\
& \left(\alpha_{e}, \beta_{e}\right): \text { Ellipsoid coordinates. }
\end{aligned}
$$

The prismatic and universal joint coordinates are now expressed in terms of the ellipsoid coordinates $\left(\alpha_{e}, \beta_{e}\right)$.

$$
\begin{aligned}
\rho & =\sqrt{\left(a_{e} \cos \left(\alpha_{e}\right) \sin \left(\beta_{e}\right)\right)^{2}+\left(b_{e} \sin \left(\alpha_{e}\right) \sin \left(\beta_{e}\right)\right)^{2}+\left(c_{e} \cos \left(\beta_{e}\right)\right)^{2}}, \\
\alpha & =\arctan \left(\frac{b_{e}}{a_{e}} \tan \left(\alpha_{e}\right)\right), \\
\beta & =\arctan \left(\sqrt{\left(a_{e} \cos \left(\alpha_{e}\right)\right)^{2}+\left(b_{e} \sin \left(\alpha_{e}\right)\right)^{2}} \tan \left(\beta_{e}\right) / c\right) .
\end{aligned}
$$

The mechanism is a universal joint with coordinates $\left(\alpha_{e}, \beta_{e}\right)$ and a passive prismatic joint with coordinate $\rho\left(\alpha_{e}, \beta_{e}\right)$.

In the kinematic shoulder model, the scapula has two contact points with the ribcage. Each contact is replaced with a mechanism described previously, yielding a kinematic model of the shoulder where the scapula is a 2-3 parallel platform. The articulations are modelled as ball and socket joints (Fig.4). There are two additional ball and socket joints at the points $T S$ and $A I$. There are two universal joints located at the centre of the ellipsoids $E$. Between the point $E$ and $T S$ there is a prismatic joint and between the point $E$ and the point $A I$ there is a another. The lengths of both prismatic joints $\rho_{T S}$ and $\rho_{A I}$ are function of the universal joint coordinates $\left(\alpha_{\mathrm{TS}}, \beta_{\mathrm{TS}}\right)$ and $\left(\alpha_{\mathrm{AI}}, \beta_{\mathrm{AI}}\right)$ according to $(20)$.

$$
\rho_{T S}=\rho_{T S}\left(\alpha_{\mathrm{TS}}, \beta_{\mathrm{TS}}\right), \quad \rho_{\mathrm{AI}}=\rho_{A I}\left(\alpha_{\mathrm{AI}}, \beta_{\mathrm{AI}}\right) .
$$

The kinematic model described in section 2 and the parallel model described in this section are equivalent. The change in representation of the scapulothoracic contact does not change the kinematic model. However, two additional kinematic chains have been added between the carrier body (thorax) and scapula. These two chains provide alternate methods of constructing a forward kinematic map. Hence the model described in this section will be referred to as the parallel shoulder model. 

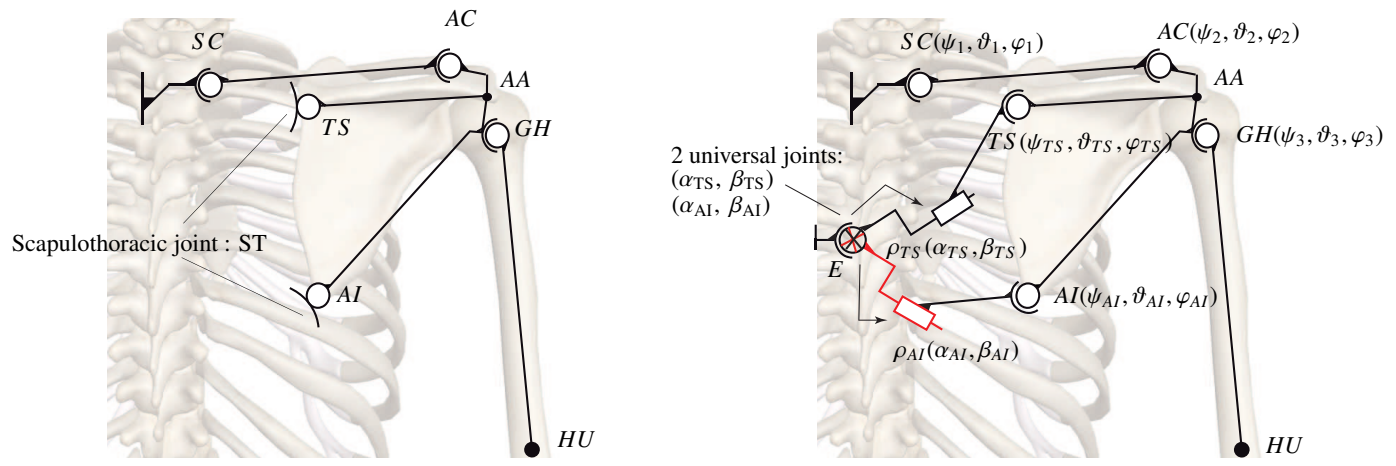

Figure 4: Illustration of the kinematic shoulder model described in section 2 and the equivalent parallel model described in section 3.

\section{Kinematic Analysis and Coordinate Reduction}

\subsection{Equivalent Kinematic Maps and Coordinates}

This section presents formal definitions of the mathematical structures involved in the kinematic shoulder models presented in sections 2 and 3, i.e. the natural and parallel models respectively. These definitions are based on the following set of assumptions: bones are defined as rigid bodies and articulations are defined as ideal mechanical joints. The shoulder model presented in section 2 is characterised by its forward kinematic map, parameterised in terms of the nine joint coordinates.

Definition 1 (The Shoulder's Natural Kinematic Map). The shoulder model's natural kinematic map is parameterised by nine coordinates, divided into three sets of three, each parameterising the configuration of a spherical kinematic pair, representing one of the three anatomical articulations. The kinematic coordinates are defined by:

$$
\begin{aligned}
& \mathscr{Q}_{S, \mathscr{N}}:=\left\{\vec{q}=\left(\begin{array}{l}
\vec{q}_{1} \\
\vec{q}_{2} \\
\vec{q}_{3}
\end{array}\right) \mid \vec{q}_{i}=\left(\begin{array}{c}
\psi_{i} \\
\vartheta_{i} \\
\varphi_{i}
\end{array}\right) \in \mathscr{Q}_{i, S} \subset[-\pi, \pi]^{3}, \phi_{i, S}\left(\mathscr{Q}_{i, S}\right) \subset S O(3), i=1,2,3\right\}, \\
& \Rightarrow \mathscr{Q}_{S, \mathscr{N}}=\mathscr{Q}_{1, S} \times \mathscr{Q}_{2, S} \times \mathscr{Q}_{3, S} \subset(S O(3))^{3} \equiv(S O(3) \times S O(3) \times S O(3)) .
\end{aligned}
$$

The end-effector is defined as the humerus and its configuration $C_{e}$ is defined as the position of the humeroulnar joint $H U$ and orientation of the humerus with respect to the inertial frame $\mathcal{R}_{0}$. Under these assumptions, such a kinematic model of the shoulder has a the following forward kinematic map:

$$
\begin{aligned}
& \Xi_{S, \mathscr{N}}: \mathscr{Q}_{S, \mathscr{N}} \rightarrow \mathscr{W}_{S, \mathscr{N}}, \\
& \vec{q} \mapsto \Xi_{S, \mathscr{N}}(\vec{q})=C_{e}, \quad \vec{\epsilon}_{0,3}=\vec{d}_{0,1}+\boldsymbol{R}_{1,0}\left(\vec{q}_{1}\right) \vec{z}_{1}+\boldsymbol{R}_{2,0}\left(\vec{q}_{2}\right) \vec{z}_{2}+\boldsymbol{R}_{3,0}\left(\vec{q}_{3}\right) \vec{\epsilon}_{3}, \\
& \text { s.t. } \quad \Phi_{T S}(\vec{q})=\left(\vec{u}_{0,2}-\vec{e}_{0}\right)^{T} \boldsymbol{E}_{T S}\left(\vec{u}_{0,2}-\vec{e}_{0}\right)-1=0, \\
& \Phi_{A I}(\vec{q})=\left(\vec{v}_{0,2}-\vec{e}_{0}\right)^{T} \boldsymbol{E}_{A I}\left(\vec{v}_{0,2}-\vec{e}_{0}\right)-1=0 .
\end{aligned}
$$

where $\vec{e}_{0}=\left(x_{e}, y_{e}, z_{e}\right)^{T}$ is the point vector of the common ellipsoid centre $E_{0}$ in the inertial frame. The map parameterises the kinematic shoulder model using nine coordinates and has seven degrees of freedom. The coordinate space $\mathscr{Q}_{S, \mathscr{N}}$ is a compact $C^{\infty}$-manifold of dimension seven. The work space $\mathscr{W}_{S, \mathscr{N}}$ is a compact $C^{\infty}$-manifold of dimension six.

$$
\mathscr{W}_{S, \mathscr{N}}:=\left\{C_{e}=\left(\begin{array}{c}
\vec{\epsilon}_{0,3} \\
\vec{q}_{3}
\end{array}\right) \in S E(3) \mid \vec{\epsilon}_{0,3} \in \mathbb{R}^{3}, \vec{q}_{3} \in \mathscr{Q}_{3, S}, C_{e}=\Xi_{S, \mathscr{N}}(\vec{q})\right\} \subset S E(3) .
$$

The parallel platform model of the shoulder replaces the scapulothoracic contact constraints by two kinematic chains, providing two alternate parameterisations of the shoulder model's kinematics. Two sets of Euler angles are 
defined for the two spherical pairs at TS and $A I:\left(\psi_{T S}, \vartheta_{T S}, \varphi_{T S}\right),\left(\psi_{A I}, \vartheta_{A I}, \varphi_{A I}\right)$ (Fig. 4). Two sets of spherical angles are defined for the two superimposed universal joints located at $E$ : $\left(\alpha_{\mathrm{TS}}, \beta_{\mathrm{TS}}\right),\left(\alpha_{\mathrm{AI}}, \beta_{\mathrm{AI}}\right)$.

The position of the humeroulnar joint centre $H U$ is defined by one of the following two expressions:

$$
\begin{array}{ll}
\text { TS map: } & \vec{\epsilon}_{0,3}=\vec{u}_{0,2}\left(\alpha_{T S}, \beta_{T S}\right)-\mathbf{R}_{2,0}\left(\vec{q}_{2}\right) \vec{u}_{2}+\mathbf{R}_{2,0}\left(\vec{q}_{2}\right) \vec{z}_{2}+\mathbf{R}_{3,0}\left(\vec{q}_{3}\right) \vec{\epsilon}_{3}, \\
\text { AI map: } & \vec{\epsilon}_{0,3}=\vec{v}_{0,2}\left(\alpha_{A I}, \beta_{A I}\right)-\mathbf{R}_{2,0}\left(\vec{q}_{2}\right) \vec{v}_{2}+\mathbf{R}_{2,0}\left(\vec{q}_{2}\right) \vec{z}_{2}+\mathbf{R}_{3,0}\left(\vec{q}_{3}\right) \vec{\epsilon}_{3},
\end{array}
$$

where the vectors $T S_{0}: \vec{u}_{0,2}\left(\alpha_{T S}, \beta_{T S}\right)$ and $A I_{0}: \vec{v}_{0,2}\left(\alpha_{A I}, \beta_{A I}\right)$ are defined by:

$$
\vec{u}_{0,2}=\left(\begin{array}{c}
a_{T S} \cos \left(\alpha_{T S}\right) \sin \left(\beta_{T S}\right)+x_{e} \\
b_{T S} \sin \left(\alpha_{T S}\right) \sin \left(\beta_{T S}\right)+y_{e} \\
c_{T S} \cos \left(\beta_{T S}\right)+z_{e}
\end{array}\right), \vec{v}_{0,2}=\left(\begin{array}{c}
a_{A I} \cos \left(\alpha_{A I}\right) \sin \left(\beta_{A I}\right)+x_{e} \\
b_{A I} \sin \left(\alpha_{A I}\right) \sin \left(\beta_{A I}\right)+y_{e} \\
c_{A I} \cos \left(\beta_{A I}\right)+z_{e}
\end{array}\right)
$$

The two definitions imply the following relation, defining an interdependency between $\left(\alpha_{T S}, \beta_{T S}\right)$ and $\left(\alpha_{A I}, \beta_{A I}\right)$.

$$
\vec{u}_{0,2}\left(\alpha_{T S}, \beta_{T S}\right)-\mathbf{R}_{2,0}\left(\vec{q}_{2}\right) \vec{u}_{2}-\vec{v}_{0,2}\left(\alpha_{A I}, \beta_{A I}\right)+\mathbf{R}_{2,0}\left(\vec{q}_{2}\right) \vec{v}_{2}=\overrightarrow{0}
$$

The two alternate definitions of $H U$ are parameterised by the following sets of coordinates:

$$
\begin{aligned}
& \hat{\vec{q}}_{T S}=\left(\begin{array}{lllllllll}
\psi_{1} & \alpha_{T S} & \beta_{T S} & \psi_{2} & \theta_{2} & \phi_{2} & \psi_{3} & \theta_{3} & \phi_{3}
\end{array}\right)^{T}, \\
& \hat{\vec{q}}_{A I}=\left(\begin{array}{lllllllll}
\psi_{1} & \alpha_{A I} & \beta_{A I} & \psi_{2} & \theta_{2} & \phi_{2} & \psi_{3} & \theta_{3} & \phi_{3}
\end{array}\right)^{T} .
\end{aligned}
$$

The coordinate $\psi_{1}$, describing the clavicle's axial rotation is not in either map (26) or (27) but is included in the coordinate set to be complete. The two alternate parameterisations are used to define two new forward kinematic maps, called the upper and lower scapulo-thoracic maps. Although different from the natural kinematic map, they parameterise the same kinematic structure.

Definition 2 (The Shoulder's Upper Scapulo-thoracic Kinematic Map). The shoulder model's upper scapulo-thoracic kinematic map uses nine kinematic coordinates defined by:

$$
\begin{aligned}
& \mathscr{Q}_{S, \mathscr{U}}:=\left\{\hat{\vec{q}}_{T S}\left(\text { eq. (30)) } \mid \psi_{1} \in \hat{\mathscr{Q}}_{1, S} \subset S^{1},\left(\alpha_{T S}, \beta_{T S}\right) \in \hat{\mathscr{Q}}_{2, S} \subset S^{2}, \vec{q}_{2} \in \mathscr{Q}_{2, S}, \vec{q}_{3} \in \mathscr{Q}_{3, S}\right\},\right. \\
& \Rightarrow \mathscr{Q}_{S, \mathscr{U}} \subset\left(S^{1} \times S^{2} \times S O(3) \times S O(3)\right) .
\end{aligned}
$$

The end-effector is defined as the humerus and its configuration $C_{e}$ is defined as the position of the humeroulnar joint $H U$ and orientation of the humerus with respect to the inertial frame $\mathcal{R}_{0}$. Under these assumptions, such a kinematic model of the shoulder has the following forward kinematic map:

$$
\begin{aligned}
\Xi_{S, \mathscr{U}}: \mathscr{Q}_{S, \mathscr{U}} & \rightarrow \mathscr{W}_{S, \mathscr{U}}, \\
\hat{\vec{q}}_{T S} & \mapsto \Xi_{S, \mathscr{U}}\left(\hat{\overrightarrow{\vec{q}}}_{T S}\right)=C_{e}, \quad \vec{\epsilon}_{0,3}=e q .(26), \\
\text { s.t. } \quad \Phi_{A C}\left(\hat{\vec{q}}_{T S}\right)=\left(\vec{d}_{0,1}-\vec{d}_{0,2}\right)^{T}\left(\vec{d}_{0,1}-\vec{d}_{0,2}\right)-\|S C-A C\|_{2}^{2}=0, & \Phi_{A I}\left(\hat{\vec{q}}_{T S}\right)=\left(\vec{v}_{0,2}-\vec{e}_{0}\right)^{T} \boldsymbol{E}_{A I}\left(\vec{v}_{0,2}-\vec{e}_{0}\right)-1=0 .
\end{aligned}
$$

where $\vec{d}_{0,1}$ and $\vec{d}_{0,2}$ are the point vectors of $S C$ and $A C$ respectively in the inertial frame. The map parameterises the kinematic shoulder model using nine coordinates and has seven degrees of freedom. The coordinate space $\mathscr{Q}_{S, \mathscr{U}}$ is compact $C^{\infty}$-manifold of dimension seven. The work space $\mathscr{W}_{S, \mathscr{U}}$ is a compact $C^{\infty}$-manifold of dimension six.

$$
\mathscr{W}_{S, \mathscr{U}}:=\left\{C_{e}=\left(\begin{array}{c}
\vec{\epsilon}_{0,3} \\
\vec{q}_{3}
\end{array}\right) \in S E(3) \mid \vec{\epsilon}_{0,3} \in \mathbb{R}^{3}, \vec{q}_{3} \in \mathscr{Q}_{3, S}, C_{e}=\Xi_{S, \mathscr{U}}(\vec{q})\right\} \subset S E(3) .
$$

Definition 3 (The Shoulder's Lower Scapulo-thoracic Kinematic Map). The shoulder model's lower scapulo-thoracic kinematic map uses nine kinematic coordinates defined by:

$$
\begin{aligned}
& \mathscr{Q}_{S, \mathscr{L}}:=\left\{\hat{\vec{q}}_{T S}(\text { eq. }(30)) \mid \psi_{1} \in \hat{\mathscr{Q}}_{1, S} \subset S^{1},\left(\alpha_{A l}, \beta_{A I}\right) \in \hat{\mathscr{Q}}_{2, S} \subset S^{2}, \vec{q}_{2} \in \mathscr{Q}_{2, S}, \vec{q}_{3} \in \mathscr{Q}_{3, S}\right\}, \\
& \Rightarrow \mathscr{Q}_{S, \mathscr{L}} \subset\left(S^{1} \times S^{2} \times S O(3) \times S O(3)\right) .
\end{aligned}
$$


The end-effector is defined as the humerus and its configuration $C_{e}$ is defined as the position of the humeroulnar joint $H U$ and orientation of the humerus with respect to the inertial frame $\mathcal{R}_{0}$. Under these assumptions, such a kinematic model of the shoulder has the following forward kinematic map, called the lower scapulothoracic kinematic map.

$$
\begin{aligned}
& \Xi_{S, \mathscr{L}}: \mathscr{Q}_{S, \mathscr{L}} \rightarrow \mathscr{W}_{S, \mathscr{L}}, \\
& \hat{\vec{q}}_{A I} \mapsto \Xi_{S, \mathscr{L}}\left(\hat{\vec{q}}_{A I}\right)=C_{e}, \vec{\epsilon}_{0,3}=e q .(27), \\
& \text { s.t. } \quad \Phi_{A C}\left(\hat{\vec{q}}_{A I}\right)=\left(\vec{d}_{0,1}-\vec{d}_{0,2}\right)^{T}\left(\vec{d}_{0,1}-\vec{d}_{0,2}\right)-\|S C-A C\|_{2}^{2}=0, \\
& \Phi_{T S}\left(\hat{\vec{q}}_{A I}\right)=\left(\vec{u}_{0,2}-\vec{e}_{0}\right)^{T} \boldsymbol{E}_{T S}\left(\vec{u}_{0,2}-\vec{e}_{0}\right)-1=0 .
\end{aligned}
$$

where $\vec{d}_{0,1}$ and $\vec{d}_{0,2}$ are the point vectors of $S C$ and $A C$ respectively in the inertial frame. The map parameterises the kinematic shoulder model using nine coordinates and has seven degrees of freedom. The coordinate space $\mathscr{Q}_{S, \mathscr{L}}$ is compact $C^{\infty}$-manifold of dimension seven. The work space $\mathscr{W}_{S, \mathscr{L}}$ is a compact $C^{\infty}$-manifold of dimension six.

$$
\mathscr{W}_{S, \mathscr{L}}:=\left\{C_{e}=\left(\begin{array}{c}
\vec{\epsilon}_{0,3} \\
\vec{q}_{3}
\end{array}\right) \in S E(3) \mid \vec{\epsilon}_{0,3} \in \mathbb{R}^{3}, \vec{q}_{3} \in \mathscr{Q}_{3, S}, C_{e}=\Xi_{S, \mathscr{L}}(\vec{q})\right\} \subset S E(3)
$$

The parallel model of the shoulder defines the shoulder girdle as a 2-3 parallel platform. The three kinematic maps presented previously define maps between a vector of nine kinematic coordinates and the configuration of the end-effector. The maps are each subject to two smooth equality constraints (Fig. 5).

(a)

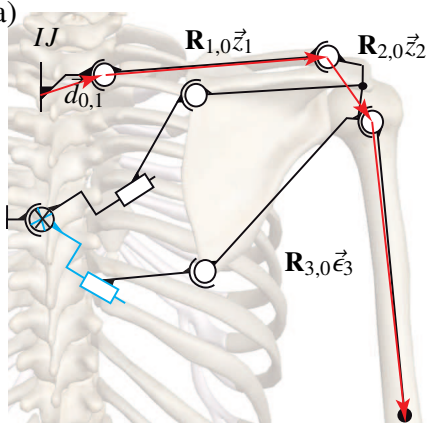

(b)

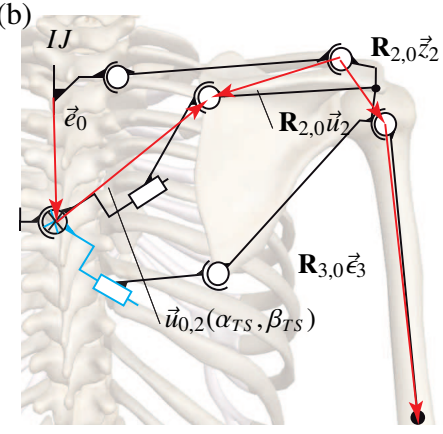

(c)

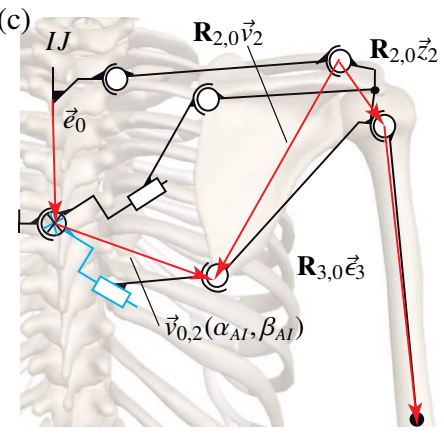

Figure 5: Illustration of the three forward kinematic maps presented in section 4.1. (a) The natural map. (b) The upper scapulo-thoracic map. (c) The lower scapulo-thoracic map.

\subsection{The Coordinate Spaces}

The coordinate spaces of all three maps $\left(\mathscr{Q}_{S, \mathscr{N}}, \mathscr{Q}_{S, \mathscr{U}}, \mathscr{Q}_{S, \mathscr{L}}\right)$ are defined as $C^{\infty}$-manifolds in $\mathbb{R}^{9}$ of dimension 7. They are all subsets of a product of $C^{\infty}$-manifolds.

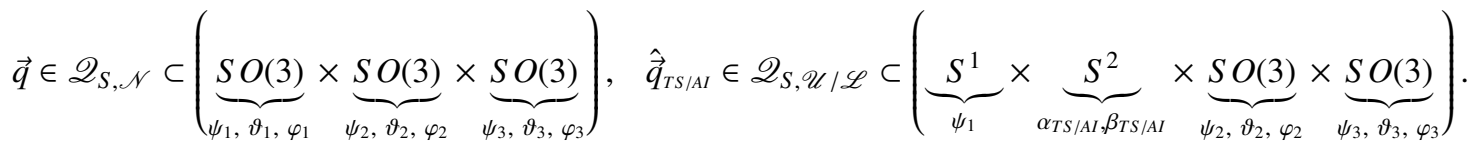

The three sets are equivalent, each parameterising the spatial configuration of three bodies that are part of the same kinematic structure. The three maps define charts from different subsets of $\mathbb{R}^{9}$ to the same subset $\mathscr{Q}_{S}$ of $(S O(3))^{3}$. Independently of the coordinates, the kinematic shoulder model's configuration evolves on a smooth manifold of dimension 7 in $\mathbb{R}^{9}$. The bodies are connected by spherical kinematic pairs and therefore, the kinematic coordinate set can always be seen as a subset of $(S O(3))^{3}$.

$$
\mathscr{Q}_{S, \mathscr{N}} \equiv \mathscr{Q}_{S, \mathscr{U}} \equiv \mathscr{Q}_{S, \mathscr{L}} \equiv \mathscr{Q}_{S} \subset(S O(3))^{3}=(S O(3) \times S O(3) \times S O(3)) .
$$




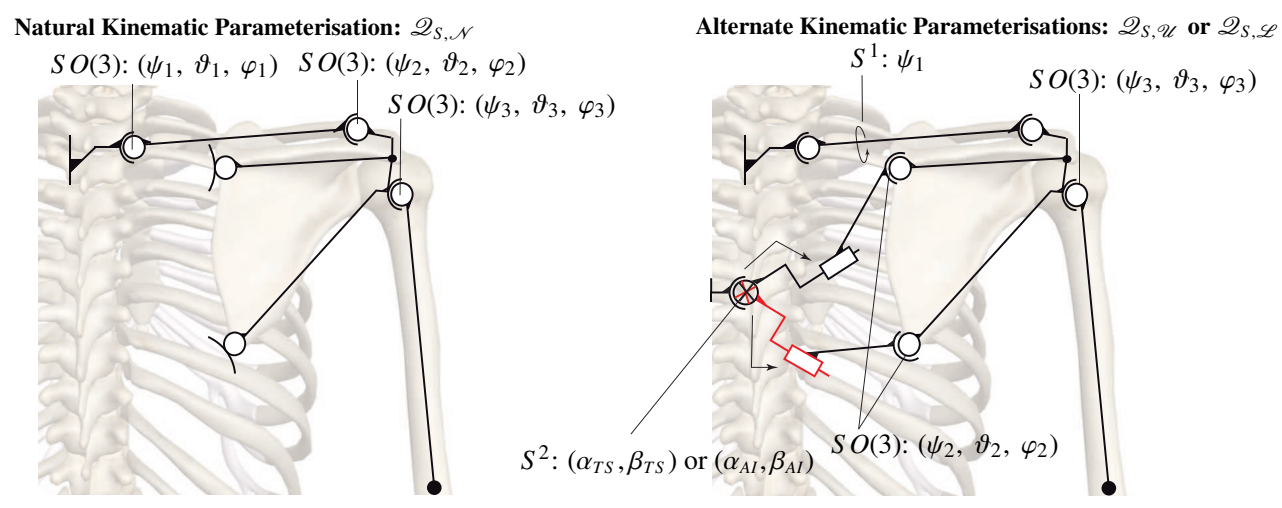

Figure 6: Illustration of the coordinates in the submanifolds as defined in section 4.2.

The natural kinematic map defines the space $\mathscr{Q}_{S}$, using three sets of Euler/Bryan angles belonging to three subsets $\mathscr{Q}_{i, S}$ of $S O(3)$. The map uses three identical charts from $[-\pi, \pi]^{3}$ to $S O(3)$ that together define the charts from $\mathbb{R}^{9}$ to the compact smooth manifold $(S O(3))^{3}$.

$$
\begin{aligned}
\phi_{i, S}:[-\pi, \pi]^{3} \subset \mathbb{R}^{3} & \rightarrow S O(3), \\
\left(\psi_{i}, \vartheta_{i}, \varphi_{i}\right) & \mapsto \phi_{i, S}\left(\psi_{i}, \vartheta_{i}, \varphi_{i}\right)=\mathbf{R}_{i, 0} .
\end{aligned}
$$

Given the constraints in the natural kinematic map, the domain spaces of the three charts $\phi_{i, S}$ are restricted to subsets $\mathscr{Q}_{i, S} \subset[-\pi, \pi]^{3} \subset \mathbb{R}^{3}$ and the image spaces are three subsets $\phi_{i, S}\left(\mathscr{Q}_{i, S}\right) \subset S O(3)$. The subsets $\mathscr{Q}_{i, S}$ are defined by the constraints. Without constraints $\mathscr{Q}_{i, S} \equiv[-\pi, \pi]^{3}$ and $\phi_{i, S}\left(\mathscr{Q}_{i, S}\right) \equiv S O(3)$ for $i=1,2,3$. The constraints are function of the clavicular and scapular coordinates only. The humeral coordinates are independent of any constraint $\mathscr{Q}_{3, S} \equiv[-\pi, \pi]^{3}$.

The upper and lower scapulo-thoracic forward kinematic maps use four maps. Two maps $\phi_{2, S}$ and $\phi_{3, S}$ from $[-\pi, \pi]^{3}$ to $S O(3)$, one map $\hat{\phi}_{1, S}$ from $[-\pi, \pi]$ to $S^{1} \equiv S O(2)$ and one map $\hat{\phi}_{2, S}$ from $[-\pi, \pi] \times\left[-\frac{\pi}{2}, \frac{\pi}{2}\right]$ to $S^{2}$. The two maps $\phi_{2, S}$ and $\phi_{3, S}$ are the same as the natural parameterisation discussed previously.

$$
\begin{aligned}
\hat{\phi}_{1, S}:[-\pi, \pi] \subset \mathbb{R} & \rightarrow S^{1}, \\
\psi_{1} & \mapsto \hat{\phi}_{1, S}\left(\psi_{1}\right)=\mathbf{R}_{x}\left(\psi_{1}\right) . \\
\hat{\phi}_{2, S}:[-\pi, \pi] \times\left[-\frac{-\pi}{2}, \frac{\pi}{2}\right] \subset \mathbb{R}^{2} & \rightarrow S^{2}, \\
\left(\alpha_{T S}, \beta_{T S}\right) \operatorname{or}\left(\alpha_{A I}, \beta_{A I}\right) & \mapsto \hat{\phi}_{2, S}\left(\alpha_{*}, \beta_{*}\right)=\mathbf{R}\left(\alpha_{*}, \beta_{*}\right) .
\end{aligned}
$$

The domain space of $\hat{\phi}_{1, S}$ is $\hat{\mathscr{Q}}_{1, S} \equiv[-\pi, \pi]$ and the image space is $\hat{\phi}_{1, S}\left(\overline{\mathscr{Q}}_{1, S}\right) \equiv S^{1} \equiv S O(2)$. The clavicle can rotate around its longitudinal axes independently of all the other coordinates. The domain space of $\hat{\phi}_{2, S}$ is a subset of the full domain space $\hat{\mathscr{Q}}_{2, S} \subset[-\pi, \pi] \times\left[-\frac{\pi}{2}, \frac{\pi}{2}\right]$ and the image space is a subset $\hat{\phi}_{2, S}\left(\hat{\mathscr{Q}}_{2, S}\right) \subset S^{2}$.

The previous analysis shows that four of the nine kinematic coordinates are independent of any constraints and of each other: $\left(\psi_{1}, \psi_{3}, \vartheta_{3}, \varphi_{3}\right)$. They define the clavicle's axial rotation and the orientation of the humerus. Furthermore, the five remaining coordinates parameterise the scapula's displacement (3). Two of the three clavicle coordinates $\left(\vartheta_{1}, \varphi_{1}\right)$ parameterise the linear position of the scapula and the three scapular coordinates $\left(\psi_{2}, \vartheta_{2}, \varphi_{2}\right)$ parameterise the orientation about the linear position.

\section{Minimal Kinematic Parameterisations}

The natural kinematic shoulder model parameterises the kinematics using nine coordinates and two equality constraints. Although the model uses nine coordinates, there are seven degrees of freedom because of the two constraints. This section presents three coordinate reductions leading to three minimal kinematic parameterisations of the shoulder 
model, each using seven independent coordinates. The coordinate reduction is based on the following observation. The upper and lower scapulothoracic kinematic maps model the scapula as a 2-3 parallel platform. The platform includes six independent kinematic pairs without including the passive prismatic pairs. The mobility formula applied to the shoulder girdle (clavicle and scapula) states that there are four degrees of freedom.

$$
D_{S}=6 \cdot 4-\sum_{i=1}^{6}\left(6-k_{i}\right)=6 \cdot 4-\underbrace{4 \cdot 3}_{\text {Spherical Joints }}-\underbrace{2 \cdot 4}_{\text {Universal Joints }}=4 .
$$

Without the clavicle's self rotation $\psi_{1}$, the scapula has three degrees of freedom. Thus far, the scapula has been parameterised by five coordinates: $\left(\theta_{1}, \phi_{1}, \psi_{2} \vartheta_{2}, \varphi_{2}\right),\left(\alpha_{T S}, \beta_{T S}, \psi_{2}, \vartheta_{2}, \varphi_{2}\right)$ or $\left(\alpha_{A I}, \beta_{A I}, \psi_{2}, \vartheta_{2}, \varphi_{2}\right)$. The three minimal sets of coordinates will use three coordinates to parameterise the configuration of the scapula and four other coordinates parameterising the clavicle's axial rotation $\psi_{1}$ and the glenohumeral Euler angles: $\left(\psi_{3}, \vartheta_{3}, \varphi_{3}\right)$.

In the parallel platform description of the shoulder girdle (thorax, clavicle and scapula), the scapula is a platform resting on three legs. It is a solid gliding on three surfaces, a sphere and two ellipsoids, through three point contacts: $A C, T S$ and $A I$. The coordinate reductions use the following principles. First, the geometric configuration of a solid is known if the position of three points on the solid are known [36]. Second, all three surfaces are quadric surfaces. Third, a single point moving on a quadric surface can be parameterised by two coordinates. Lastly, the intersection of two quadrics can be parameterised by a single coordinate [37, 38]. These four principles are used to obtain three coordinates parameterising the scapula's configuration, thereby eliminating two of the five coordinates previously listed.

The parallel model defines the points $T S$ and $A I$ as articulations. Its configuration is defined using six polynomial equations. The polynomials are defined in terms of the cartesian coordinates of the three articulation points $A C, T S, A I$.

TS ellipsoid:

AI ellipsoid:

AC is on a sphere around SC: $\quad\left(\vec{d}_{0,2}-\vec{d}_{0,1}\right)^{T}\left(\vec{d}_{0,2}-\vec{d}_{0,1}\right)=\rho_{\mathrm{AC}}^{2}$,

TS is on a sphere around AC: $\quad\left(\vec{d}_{0,2}-\vec{u}_{0,2}\right)^{T}\left(\vec{d}_{0,2}-\vec{u}_{0,2}\right)=\mu_{1}^{2}$,

AI is on a sphere around AC: $\quad\left(\vec{d}_{0,2}-\vec{v}_{0,2}\right)^{T}\left(\vec{d}_{0,2}-\vec{v}_{0,2}\right)=\mu_{2}^{2}$,

TS is on a sphere around AI: $\quad\left(\vec{u}_{0,2}-\vec{v}_{0,2}\right)^{T}\left(\vec{u}_{0,2}-\vec{v}_{0,2}\right)=\mu_{3}^{2}$.

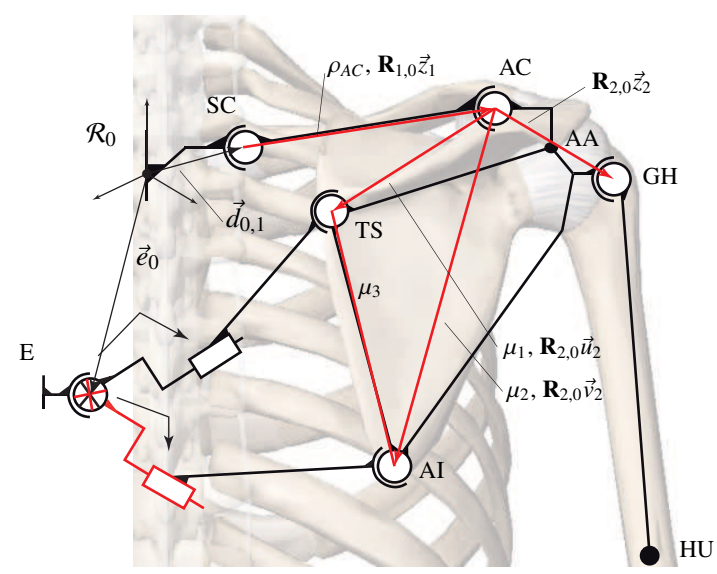

$\mathbf{R}_{2,0} \vec{z}_{2}$

SC point vector: $\vec{d}_{0,1}$,

AC point vector: $\vec{d}_{0,2}=\vec{d}_{0,1}+\mathbf{R}_{1,0} \vec{z}_{1}$,

TS point vector: $\vec{u}_{0,2}=\vec{d}_{0,1}+\mathbf{R}_{1,0} \vec{z}_{1}+\mathbf{R}_{2,0} \vec{u}_{2}$,

AI point vector: $\vec{v}_{0,2}=\vec{d}_{0,1}+\mathbf{R}_{1,0} \vec{z}_{1}+\mathbf{R}_{2,0} \vec{v}_{2}$,

E point vector: $\vec{e}_{0}$

Figure 7: Diagram illustrating the vectors and constants defined in the polynomial description of the scapula's configuration (47)-(52). 
The first three equations define the location of the three points with respect to the thorax and the remaining equations define their position with respect to each other (Fig. 7). Applying the first principle stated previously means that the scapula's configuration is known if the locations of the points $A C, T S$ and $A I$ are known. Once these points are known, any point on the scapula, for instance $G H_{0,2}$, is defined by:

$$
G H_{0,2}: \vec{z}_{2,0}=\vec{d}_{0,2}+\mathbf{R}_{2,0} \vec{z}_{2}
$$

The scapula has three degrees of freedom. Three coordinates are needed to define the position of the scapula. All six polynomial equations stated previously define quadrics. Using the second and third principles, each of the three points lies on a quadric surface, parameterised by two coordinates.

- AC: $\vartheta_{1}, \varphi_{1}$, the $y$ - and $z$-axis Bryan angles,

- TS: $\alpha_{T S}, \beta_{T S}$, the two spherical coordinates of the TS ellipsoid,

- AI: $\alpha_{A l}, \beta_{A l}$, the two spherical coordinates of the AI ellipsoid.

Once the position of either of these points is known using the pairs $\left(\vartheta_{1}, \varphi_{1}\right),\left(\alpha_{T S}, \beta_{T S}\right)$ or $\left(\alpha_{A I}, \beta_{A I}\right)$ respectively, the scapula has a single remaining degree of freedom $\xi_{S}$. The coordinate defines the motion of one of the other two points with respect to the one that is known. The point in question lies on the intersection of two quadrics. Using the fourth principle, the parameterisation of the intersection between two quadrics is well defined and is extensively used in computer aided design software (CAD). The locus can be parameterised using a single coordinate $\xi_{S}$ [37, 38]. Based on this analysis, three sets of minimal coordinates are defined. Once two of the three points on the scapula are known, the last point lies on the intersection between three quadric surfaces, the locus of which is a point.

Definition 4 (Natural Minimal Coordinates). The natural kinematic map is parameterised in terms of the following vector of minimal coordinates:

$$
\vec{q}^{*}=\left(\psi_{1}, \vartheta_{1}, \varphi_{1}, \xi_{S}, \psi_{3}, \vartheta_{3}, \varphi_{3}\right)^{T} \in \mathscr{Q}_{S, \mathscr{N}}^{*} \subset\left(S O(3) \times S^{1} \times S O(3)\right),
$$

where $\xi_{S}$ parameterises the intersection between the sphere centred at AC and either of the two ellipsoids centered at E.

Definition 5 (Upper Scapulothoracic Minimal Coordinates). The upper scapulothoracic kinematic map defines the following vector of minimal coordinates:

$$
\vec{q}^{*}=\left(\psi_{1}, \alpha_{T S}, \beta_{T S}, \xi_{S}, \psi_{3}, \vartheta_{3}, \varphi_{3}\right)^{T} \in \mathscr{Q}_{S, \mathscr{U}}^{*} \subset\left(S^{1} \times S^{2} \times S^{1} \times S O(3)\right)
$$

where $\xi_{S}$ parameterises the intersection between the sphere centred at TS and the ellipsoid centered at AI or the sphere centered at $S C$.

Definition 6 (Lower Scapulothoracic Minimal Coordinates). The lower scapulothoracic kinematic map defines the following vector of minimal coordinates:

$$
\vec{q}^{*}=\left(\psi_{1}, \alpha_{A I}, \beta_{A I}, \xi_{S}, \psi_{3}, \vartheta_{3}, \varphi_{3}\right)^{T} \in \mathscr{Q}_{S, \mathscr{L}}^{*} \subset\left(S^{1} \times S^{2} \times S^{1} \times S O(3)\right)
$$

where $\xi_{S}$ parameterises the intersection between the sphere centred at AI and the ellipsoid centered at TS or the sphere centered at $S C$.

In all three definitions, $\xi_{S}$ parameterises the intersection between a sphere and an ellipsoid and is not an angular coordinate. The intersection locus is homeomorphic to a circle and $\xi_{S}$ has an associated chart between $[0,1] \subset \mathbb{R}$ and $S^{1}$.

$$
\begin{aligned}
\phi_{2, S}^{*}:[0,1] \subset \mathbb{R} & \rightarrow S^{1} \\
\xi_{S} & \mapsto \phi_{2, S}^{*}\left(\xi_{S}\right)=\mathbf{R}_{2,0}\left(\xi_{S}\right)
\end{aligned}
$$


The domain space is restricted to $\mathscr{Q}_{2, S}^{*} \subset[0,1]$ because the triple sphere intersection, defining the position of the third point on the scapula, must exist. Given the location of two of the three points, the third point must lie on the intersection of three spheres. This constraint limits the actual domain space and the image space is therefore also a subset $\phi_{2, S}^{*}\left(\mathscr{Q}_{2, S}^{*}\right) \subset S^{1}$. This condition also restricts the domain space of the chart associated to $\left(\vartheta_{1}, \varphi_{1}\right),\left(\alpha_{T S}, \beta_{T S}\right)$ or $\left(\alpha_{A I}, \beta_{A I}\right)$, depending on the set of minimal coordinates.

The coordinate space $\mathscr{Q}_{S}$ of the shoulder model is defined by a set of seven coordinates ((54), (55) or (56)). The set of seven coordinates is said to be a minimal set of coordinates in the sense that the number of coordinates is equal to the number of degrees of freedom. There are three possible choices of coordinates depending on which kinematic chain is used. The coordinates define maps from subsets of $\mathbb{R}^{7}$ to the kinematic coordinate manifold, submanifold of $(S O(3))^{3}$.
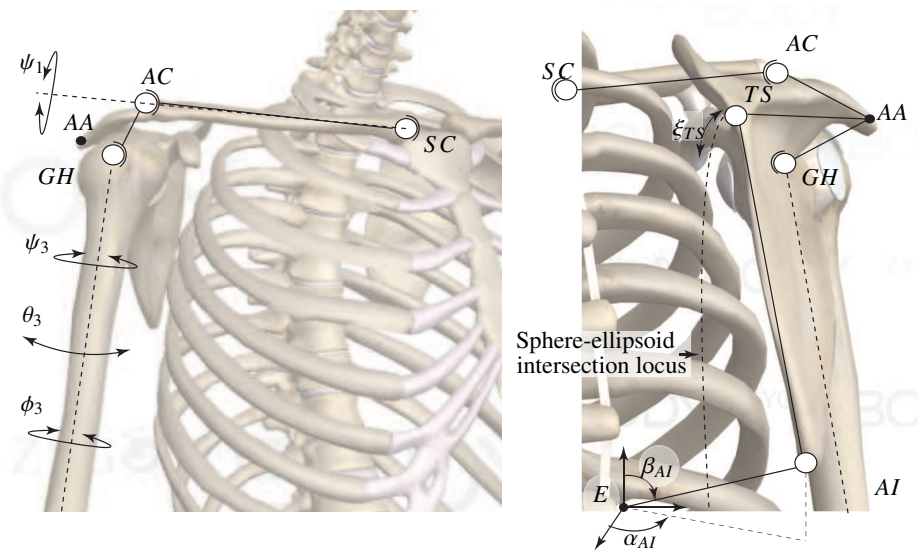

Figure 8: Illustration of the minimal set of coordinates, using the lower scapulo-thoracic kinematic map as described in section 5. Image created using ZygoteBody ${ }^{T M}$ zygotebody.com.

The chart associated to the minimal coordinates, from a subset of $\mathbb{R}^{7}$ onto the coordinate manifold $\mathscr{Q}_{S}$, using the natural kinematic map, is defined by:

$$
\begin{aligned}
\phi_{S, \mathscr{N}}: \mathscr{Q}_{1, S} \times \mathscr{Q}_{2, S}^{*} \times \mathscr{Q}_{3, S} & \rightarrow \mathscr{Q}_{S}, \\
\vec{q}^{*}=\left(\psi_{1}, \vartheta_{1}, \varphi_{1}, \xi_{S}, \psi_{3}, \vartheta_{3}, \varphi_{3}\right) & \mapsto \phi_{S, \mathscr{N}}\left(\vec{q}^{*}\right)=\left(\phi_{1, S}\left(\psi_{1}, \vartheta_{1}, \varphi_{1}\right), \phi_{2, S}^{*}\left(\xi_{S}\right), \phi_{3, S}\left(\psi_{3}, \vartheta_{3}, \varphi_{3}\right)\right)=\left(\mathbf{R}_{1,0}, \mathbf{R}_{2,0}, \mathbf{R}_{3,0}\right) .
\end{aligned}
$$

The charts associated to the minimal coordinates, constructed from the upper/lower scapulo-thoracic kinematic map, are defined by:

$$
\begin{aligned}
\phi_{S, \mathscr{U}}: \hat{\mathscr{Q}}_{1, S} \times \hat{\mathscr{Q}}_{2, S} \times \mathscr{Q}_{2, S}^{*} \times \mathscr{Q}_{3, S} & \rightarrow \mathscr{Q}_{S}, \\
\vec{q}^{*}=\left(\psi_{1}, \alpha_{T S}, \beta_{T S}, \xi_{S}, \psi_{3}, \vartheta_{3}, \varphi_{3}\right) & \mapsto \phi_{S, \mathscr{U}}\left(\vec{q}^{*}\right)=\left(\hat{\phi}_{1, S}\left(\psi_{1}\right), \hat{\phi}_{2, S}\left(\alpha_{T S}, \beta_{T S}\right), \phi_{2, S}^{*}\left(\xi_{S}\right), \phi_{3, S}\left(\psi_{3}, \vartheta_{3}, \varphi_{3}\right)\right)=\left(\mathbf{R}_{1,0}, \mathbf{R}_{2,0}, \mathbf{R}_{3,0}\right) . \\
\phi_{S, \mathscr{L}}: \hat{\mathscr{Q}}_{1, S} \times \hat{\mathscr{Q}}_{2, S} \times \mathscr{Q}_{2, S}^{*} \times \mathscr{Q}_{3, S} & \rightarrow \mathscr{Q}_{S}, \\
\vec{q}^{*}=\left(\psi_{1}, \alpha_{A l}, \beta_{A l}, \xi_{S}, \psi_{3}, \vartheta_{3}, \varphi_{3}\right) & \mapsto \phi_{S, \mathscr{L}}\left(\vec{q}^{*}\right)=\left(\hat{\phi}_{1, S}\left(\psi_{1}\right), \hat{\phi}_{2, S}\left(\alpha_{A l}, \beta_{A I}\right), \phi_{2, S}^{*}\left(\xi_{S}\right), \phi_{3, S}\left(\psi_{3}, \vartheta_{3}, \varphi_{3}\right)\right)=\left(\mathbf{R}_{1,0}, \mathbf{R}_{2,0}, \mathbf{R}_{3,0}\right) .
\end{aligned}
$$

In comparison to each other, the three possible sets of minimal coordinate parameterise the same kinematic structure. Given this point, it is natural to ask if they are exactly equivalent. The short answer is no. They are similar but not identical. There are four coordinates that are found in all three sets of minimal coordinates. The differences between the sets lie in the three coordinates parameterising the scapula's configuration. For instance, if the natural set of minimal coordinates is used, the third point on the scapula (TS or AI) is obtained by computing the intersection between two spheres and an ellipsoid. For the other two sets of minimal coordinates, the third point is AC, lying on the common intersection is between three spheres. Finally, an important difference between the coordinate sets is 
in their implementation. The numerical range of each coordinate will differ. Furthermore, this paper presented the definition of the three coordinate sets without discussing the numerical values. The specifics of the domain space of the kinematic maps will depend on the numerical values of the shoulder model. It could be that when implemented, a coordinate set could lead to difficulties because of numerical problems. The most likely source of trouble is the definition of the ellipsoids. Their orientation and dimensions will have an impact on the implementation.

\section{Discussion}

This paper presented a kinematic parallel model of the shoulder. The scapulothoracic contact, is modelled in this paper using kinematic chains. The parallel model is kinematically equivalent to a model using constraints. A kinematic analysis of the parallel model lead to the construction of three minimal parameterisations.

Many musculoskeletal shoulder models parameterise the kinematics of the shoulder using nine joint coordinates which have a physiological meaning $[14,7,1,10,11,2]$. The scapulothoracic contact is then modelled using constraints $[7,1,10,11,8]$, given that it is not a joint in the anatomical sense. The constraints reduce the number of degrees of freedom but add complexity to the model. As stated in the introduction, kinematic redundancy means that there are an infinite number of internal joint configurations, yielding the same end-effector (elbow) configuration. The challenge of redundancy is to select appropriate internal joint configurations. In robotics, solutions are selected according to the requirements of the robot. For instance to use less energy. In musculoskeletal modelling, the kinematics must reflect reality, which makes motion planning in musculoskeletal shoulder models particularly challenging. Without considering constraints, the nine joint coordinates are independent and measured data (measured joint angles) can be directly used in the model. The model's joint angles can be assigned the values of the measured angles. However, not considering the scapulothoracic contact limits the validity of the model. With constraints, the nine coordinates become interdependent and motion planning requires using numerical algorithms to find solutions that follow measured motion while respecting the constraints $[39,40,12,13]$. This point is what makes the minimal parameterisations attractive. The minimal coordinates presented in this paper are independent from each other and incorporate the constraints. If measured motion is defined in terms of the minimal coordinates, it can be directly applied to the model. Motion planning in terms of the minimal coordinates is considerably simplified. However, the minimal coordinates are not all joint coordinates. They do not all have a direct physiological significance. Furthermore, it is very hard to describe the model's dynamics in terms of these coordinates. The minimal coordinates are a purely kinematic tool. They allow one to easily plan motion. Once planned, the motion is transformed to obtain the joint angle parameterisation. A dynamic model built from the joint angle parameterisation is then used to predict the kinetics.

Another limitation of the minimal coordinates is that they parameterise a normal shoulder. The minimal coordinates as presented in this paper cannot parameterise a pathological shoulder. For instance, the "winging" scapula behaviour could not be obtained using the minimal coordinates as presented in this paper. However, the passive prismatic joint $\left(\rho_{\alpha}, \rho_{\beta}\right)$ could be modified to allow the scapula to lift off the surface of the ribcage. The minimal coordinates in their current form can only simulate healthy shoulder movement but could be adapted to incorporate pathological behaviours. Again, this would only be in a purely kinematic context. All motion planned in terms of the minimal coordinates would need to be mapped back to a joint angle parameterisation.

A last point concerning the parallel model and its use in musculoskeletal modelling concerns the kinematic chains. The parallel model of the shoulder presented in this paper uses kinematic chains to model the scapulothoracic contact. Like the minimal coordinates, these chains are kinematic and have no meaning beyond the context of motion planning. The scapula can only push against the ribcage, there is only a compressive force. If put in a dynamic context, the kinematic chains defined in this paper would be capable of a compressive and tensile force. Thus, the minimal coordinates simplify motion planning but remain a purely kinematic tool. Shoulder dynamics should be parameterised in terms of joint angles to avoid such physical inconsistencies.

The minimal coordinates also have an application in more general parallel mechanisms. Indeed, the minimal coordinates presented in this paper are based on the philosophy of parameterising the intersections between surfaces. In other parallel platforms, like the Gough-Stewart platform [41, 42] there are only prismatic and universal joints. Thus, all the points on the platform lie on spheres centred around the base points. A minimal set of coordinates, like the ones presented here, could be built which would simplify motion planning. However, such an application would require 
further investigation.

The comparison of the shoulder skeletal structure to the Stewart platform leads to the natural question of whether a mechanical system can be constructed with identical or similar kinematic characteristics to that of the shoulder? A possible feasible mechanism is obtained by separating the two universal joints, located at $E$ (Fig. 8). The altered mechanism would have two universal joints located at points $E_{1}$ and $E_{2}$. Indeed, the parallel shoulder mechanism is not directly a possible solution because of the superimposed universal joints. The change in joint location does not alter the kinematic properties of the system. Indeed, the kinematics are still defined by a set of polynomial equations ((47)-(52)) with the difference that one of the ellipsoid equations is translated away from the origin. Also, the minimal coordinate construction method remains the same. It is of course not necessary to impose the ellipsoid nature of the scapulothoracic gliding plane on the length of the legs $E_{1}-T S$ and $E_{2}-A I$. One could impose spherical behaviour or even planar behaviour. This would considerably simplify the description of the platform's motion. In both cases, the point $T S$ would be described with respect to $A I$, or vice versa, using an intersection coordinate $\xi_{S}$. For spherical behaviour, $T S$ moves on the intersection of two spheres which is a circle. For planar behaviour, $T S$ moves in a circle around $A I$. Before a physical implementation of the system is considered, it would be necessary to investigate their kinematics in detail to locate any possible singular points and whether or not the control is actually possible. Additionally, the structural properties of such systems should also require attention.

Finally, the concept of replacing a contact constraint with a kinematic chains could be generalised. Indeed, the ideas presented in this paper could form the basis of a general methodology of constructing alternate parameterisations of kinematic models containing contact constraints. Furthermore, Gröbner bases could be used to reduce the polynomial description of the scapula's configuration in section $5[43,44]$.

\section{Conclusions}

The shoulder's kinematics are challenging to replicate in models and real mechanisms because of the complexity of the contact between the scapula and thorax. Therefore, the goal of this paper was to present a novel parallel model of the shoulder without constraints, thereby simplifying the task of motion planning in models and mechanically replacing the gliding architecture with a parallel architecture. The model is built on an anatomically accurate and mathematically rigorous analysis of a shoulder model's kinematics from the literature. The presentation focuses on the contact that is usually represented using equality constraints. This work uses the novel idea, with respect to the shoulder, of replacing the contact constraints with kinematic chains. A rigorous analysis of the shoulder's kinematics was also presented, resulting in three minimal kinematic parameterisations without constraints. The parameterisations are not strictly equivalent but parameterise the same underlying kinematic structure. The minimal parameterisations use a number of independent coordinates equal to the number of degrees of freedom. The coordinates represent an efficient method of planing motion for the model and the parallel architecture is mechanically simpler to implement, than the gliding architecture.

\section{Conflict of interest statement}

None of the authors has any conflict of interest.

\section{Acknowledgements}

This study was supported by the Swiss National Science Foundation (K-32K1-122512).

\section{References}

[1] B. Garner, M. Pandy, A kinematic model of the upper limb based on the visible human project (vhp) image dataset, Computer Methods in Biomechanics and Biomedical Engineering 2 (2) (2001) 93-126.

[2] I. Charlton, G. Johnson, A model for the prediction of the forces at the glenohumeral joint, in: Proc. Inst. Mech. Eng. [H], Vol. 220, 2006, p. 801-812. 
[3] A. Nikooyan, H. Veeger, P. Westerhoff, F. Graichen, G. Bergmann, F. van der Helm, Validation of the delft shoulder and elbow model using in-vivo glenohumeral joint contact forces, Journal of Biomechanics 43 (15) (2010) 3007 - 3014

[4] M. Okada, Y. Nakamura, Development of a cybernetic shoulder-a 3-dof mechanism that imitates biological shoulder motion, Robotics, IEEE Transactions on 21 (3) (1999) $438-444$.

[5] M. Stanisic, J. Wiitala, J. Feix, A dexterous humanoid shoulder mechanism, Journal of Robotic Systems 18 (2) (2001) $737-745$.

[6] J. Lenarcic, M. Stanisic, A humanoid shoulder complex and the humeral pointing kinematics, Robotics and Automation, IEEE Transactions on 19 (3) (2003) $499-506$.

[7] F. van der Helm, Analysis of the kinematic and dynamic behaviour of the shoulder mechanism, Journal of Biomechanics 27 (5) (1994) 527 550.

[8] A. El Habachi, S. Duprey, L. Cheze, R. Dumas, A parallel mechanism of the shoulder-application to multi-body optimisation, Multibody System Dynamics (2014) 1-13.

[9] O. Khatib, E. Demircan, V. De Sapio, L. Sentis, T. Besier, S. Delp, Robotics-based synthesis of human motion, Journal of Physiology 103 (2009) 211-219.

[10] K. R. S. Holzbaur, W. M. Murray, S. L. Delp, A model of the upper extremity for simulating musculoskeletal surgery and analyzing neuromuscular control, Annals of Biomedical Engineering 33 (2005) 829-840.

[11] M. Damsgaard, J. Rasmussen, S. Christensen, E. Surma, M. de Zee, Analysis of musculoskeletal systems in the anybody modeling system, Simulation Modelling Practice and Theory 14 (8) (2006) 1100 - 1111.

[12] A. Nikooyan, H. Veeger, E. Chadwick, M. Praagman, F. van der Helm, Development of a comprehensive musculoskeletal model of the shoulder and elbow, Journal of Biomechanics 43 (25) (2011) 3007 - 3014.

[13] B. Bolsterlee, H. Veeger, F. van der Helm, Modelling clavicular and scapular kinematics: from measurement to simulation, Medical \& Biological Engineering \& Computing 52 (3) (2014) 283-291.

[14] C. Högfors, B. Peterson, G. Sigholm, P. Herberts, Biomechanical model of the human shoulder-ii. the shoulder ryrthm, Journal of biomechanics 24 (8) (1991) 699-709.

[15] J. H. deGroot, R. Brand, A three-dimension regression model of the shoulder rhythm, Clinical biomechanics 16 (2001) 735-743.

[16] X. Xu, J. Lin, R. W. McGorry, A regression-based 3-d shoulder rhythm, Journal of Biomechanics In Press (2014) -.

[17] A. Karduna, P. McClure, L. Michener, B. Sennett, Dynamic measurements of three-dimensional scapular kinematics: A validation study, Journal of Biomechanical Engineering 123 (2) (2001) 184-190.

[18] D. Bourne, A. Choo, W. Regan, D. MacIntyre, T. Oxland, Three-dimensional rotation of the scapula during functional movements: An in vivo study in healthy volunteers, Journal of Shoulder and Elbow Surgery 16 (2) (2007) 150 - 162.

[19] M. Rosheim, In the footsteps of leonardo articulated anthropomorphic robot, Robotics Automation Magazine, IEEE 4 (2) (1997) $12-14$.

[20] Y. Sodeyama, I. Mizuuchi, T. Yoshikai, Y. Nakanishi, M. Inaba, A shoulder structure of muscle-driven humanoid with shoulder blades, in: Intelligent Robots and Systems. (IROS 2005). 2005 IEEE/RSJ International Conference on, 2005, pp. 4028 - 4033.

[21] S. Ikemoto, F. Kannou, K. Hosoda, Humanlike shoulder complex for musculoskeletal robot arms, in: Intelligent Robots and Systems (IROS), 2012 IEEE/RSJ International Conference on, 2012, pp. 4892 - 4897.

[22] T. Kozuki, Y. Motegi, T. Shirai, Y. Asano, J. Urata, Y. Nakanishi, K. Okada, M. Inaba, Design of upper limb by adhesion of muscles and bones - detail human mimetic musculoskeletal humanoid kenshiro, in: Intelligent Robots and Systems (IROS), 2013 IEEE/RSJ International Conference on, 2013, pp. 935-940.

[23] V. Inman, M. Saunders, L. a. Abbott, The role of the scapula in athletic shoulder function. 1944, Clinical Orthopaedics Related Research 330 (1996) 3-12.

[24] D. Ingram, C. Engelhardt, A. Farron, A. Terrier, P. Mullhaupt, A minimal set of coordinates for describing humanoid shoulder motion, in: Intelligent Robots and Systems (IROS), 2013 IEEE/RSJ International Conference on, 2013, pp. 5537-5544.

[25] G. Wu, F. van der Helm, H. Veeger, M. Makhsous, P. Van Roy, C. Anglin, J. Nagels, A. Karduna, K. McQuade, X. Wang, F. Werner, B. Buchholz, Isb recommendation on definitions of joint coordinate systems of various joints for the reporting of human joint motion-part ii: shoulder, elbow, wrist and hand, Journal of Biomechanics 38 (5) (2005) $981-992$.

[26] F. van der Helm, A finite element musculoskeletal model of the shoulder mechanism, Journal of Biomechanics 27 (5) (1994) 551 - 553, $555-569$.

[27] J. Burdick, Kinematic analysis and design of redundant robot manipulators, Ph.D. thesis, Standford University, Standford (1988).

[28] E. Conkur, R. Buckingham, Clarifying the definition of redundancy as used in robotics, Robotica 15 (1997) 583 - 586.

[29] J. Uicker, G. Pennock, J. Shigley, Theory of Machines and Mechanisms, Oxford University Press, New York, 2003.

[30] B. Siciliano, O. Khatib, Springer Handbood of Robotics, Srpinger Handbooks., 2008

[31] M. Savage, Four-link mechanisms with cylindric, revolute and prismatic pairs, Mechanism and Machine Theory 7 (2) (1972) 191 - 208.

[32] J. Yuh, T. Young, Y. Baek, Modeling of a flexible link having a prismatic joint in robot mechanism-experimental verification, in: Robotics and Automation, 1989. Proceedings., 1989 IEEE International Conference on, 1989, pp. 722-727 vol.2.

[33] O. Bottema, R. B., Theoretical Kinematics, Dover Publications, 2012.

[34] S. R. Hummel, C. Chassapis, Configuration design and optimization of universal joints with manufacturing tolerances, Mechanism and Machine Theory 35 (3) (2000) $463-476$

[35] G. Zhang, J. Du, S. To, Study of the workspace of a class of universal joints, Mechanism and Machine Theory 73 (0) (2014) 244 - 258.

[36] L. Chèze, B. Fregly, J. Dimnet, A solidification procedure to facilitate kinematic analyses based on video system data, Journal of Biomechanics 28 (7) (1995) $879-884$

[37] J. Levin, A parametric algorithm for drawing pictures of solid objects composed of quadric surfaces, Commun. ACM 19 (10) (1976) 555-563.

[38] J. Levin, Mathematical models for determining the intersections of quadric surfaces, Computer Graphics and Image Processing 11 (1) (1979) $73-87$.

[39] A. Erdemir, S. McLean, W. Herzog, A. van den Bogert, Model-based estimation of muscle forces exerted during movements, Clinical Biomechanics 22 (2007) 131-154.

[40] M. Andersen, M. Damsgaard, J. Rasmussen, Kinematic analysis of over-determinate biomechanical systems, Computer Methods in Biome- 
chanics and Biomedical Engineering 12 (4) (2009) 371-384.

[41] V. Gough, S. Whitehall, Universal tyre test machine, in: Proceedings of 9th. Int. Congress of F.I.S.I.T.A, 117, 1962 , p. 117-135.

[42] D. Stewart, A platform with six degrees of freedom, in: Proceedings of the Institute of Mechanical Engineering, London, UK, Vol. 180, 1965, p. 371-386.

[43] B. Buchberger, Groebner bases and systems theory, Multidimensional Syst. Signal Process. 12 (3-4) (2001) $223-251$.

[44] D. Cox, J. Little, D. O'Shea, Ideals, Varieties, and Algorithms, New-York, Springer, 2007.

[45] J. Selig, Geometrical Methods in Robotics, Springer-Verlag, 1996.

[46] M. Spivak, A Comprehensive Introduction to Differential Geometry, 3rd Edition, Publish or Perish, 2005.

[47] R. Godement, Introduction à la théorie des goupes de Lie, Springer-Verlag, 2004.

[48] N. Bourbaki, Eléments de Mathématique, Algèbre, Chapitres 1 à 3, Diffusion C.C.L.S, Paris, 1970.

[49] N. Bourbaki, Eléments de Mathématique, Groupes et Algèbres de Lie, Chapitres 2 et 3, Hermann, Paris, 1972.

[50] C. Chevalley, Theory of Lie Groups, Princeton University Press, 1946.

[51] H. Weyl, The Classical Groups: Their Invariants and Representations, Princeton University Press, Princeton, New Jersey, 1939.

[52] I. Gelfand, R. Minlos, Z. Shapiro, Representations of the Rotation and Lorentz Groups an their Applications, Pergamon Press, New York, 1963.

[53] J. Hervé, Analyse structurelle de mécanismes par groupe des déplacements, Mechanism and Machine Theory 13 (1978) 437-450.

\section{Appendix A. Lie Group Formalism Applied to Mechanical Displacements}

In the paper we have used the following symbols $\mathcal{S}^{1}, \mathcal{S}^{2}, S O(3)$ and $S E(3)$. This short appendix provides a description of these symbols together with some relevant literature. For instance, [45] is our main reference on these topics which focuses on robotics as a background and perspective.

\section{Appendix A.1. Manifolds}

Informally, a manifold is a set that locally resembles euclidean space but globally it does not. To achieve a rigourous definition, one constitute a set of maps from the initial manifold $\mathcal{M}$ to some subset of euclidean space $\mathbb{R}^{n}$. The integer $n$ then denotes the dimension of the manifold. Each map (in the set of maps) maps different parts of $\mathcal{M}$ in such a way as to cover $\mathcal{M}$ completely. In general, there is at least two different maps in the set (which is called an atlas) and they must satisfy compatibility conditions among them (so that the definition is consistent, see for instance [46] or [47]). The following symbols appearing in the paper $\mathcal{S}^{1}, \mathcal{S}^{2}, S O(3)$ and $S E(3)$ are all examples of manifolds $\mathcal{M}$. The manifold $\mathcal{S}^{1}$ stands for the circle and $\mathcal{S}^{2}$ stands for the surface of the sphere. The integers 1 and 2 in these symbols denote the respective dimension of the Euclidean space to which the manifold locally corresponds to. The word 'atlas' can then be seen (in the case of $\mathcal{S}^{2}$ ) to correspond to its natural meaning. Concerning $S O(3)$ and $S E(3)$, they are both manifolds, $S O(3)$ is of dimension 3 and $S E(3)$ is of dimension 6 . They however have more structure than our previous two examples, i.e. they share the structure of a group.

Appendix A.2. Groups, Subgroups and Semi-Direct Products

A group is a set $G$ with binary operation $\top: G \times G \rightarrow G$ which is associative

$$
\left(g_{1} \top g_{2}\right) \top g_{3}=g_{1} \top\left(g_{2} \top g_{3}\right) \quad \forall g_{1}, g_{2} \in G,
$$

with a neutral element $e \in G$ such that

$$
g \top e=e \top g \quad \forall g \in G,
$$

and such that every $g$ has an inverse $g^{-1}$, i.e. an element for which

$$
g \top g^{-1}=e .
$$

A subgroup $H \subseteq G$ is a subset of $H$ which is closed with respect to $T$. It is then possible to define a product between two separate groups $G$ and $J$ once an homomorphism $\tau$ of $J$ into the endomorphism of $G$ is given, i.e. $\tau: J \rightarrow \operatorname{End}(G)$. It is the semi-direct product [48] of $G$ and $J$ written as

$$
G \ltimes_{\tau} J
$$

or $G \ltimes J$ when $\tau$ is obvious from the context, and is defined as

$$
\left(g_{1}, j_{1}\right) \top\left(g_{2}, j_{2}\right)=\left(g_{1} \top^{(1)} \tau\left(j_{1}\right)\left(g_{2}\right), j_{1} \top^{(2)} j_{2}\right) \quad \forall g_{1}, g_{2} \in G \quad \forall j_{1}, j_{2} \in J,
$$


where $T^{(1)}$ is the group operation of $G$ and $T^{(2)}$ is the group operation of $J$. Obviously we can reverse the symbol

$$
J \rtimes G,
$$

to denote $G \ltimes J$ which is often used in robotics text (for example in [45]).

\section{Appendix A.3. Lie Groups}

A lie group is the combination of both a manifold and a group. Additionnally it is sometimes required that the manifold be analytic (the maps to the corresponding euclidean space should be analytic [49], Ch. III, p. 96 Definition 1 , for example or [50], but it is not always the case).

\section{Appendix A.3.1. $\mathrm{SO}(3)$}

$S O(3)$ is the group of orthogonal matrices, a subset of matrices $M \in \mathbb{R}^{3 \times 3}$ having 1 as determinant, that is, all columns or rows are orthogonal and $|M|=1$ for $M \in S O(3)$. Note that this is the original definition of $S O(3)$ as a special subgroup of matrices which is a group among the classical groups [51] linked to specific transformation of space. Its classification and study as a Lie group as such together with the relation with its Lie algebra was undertaken later (for example [50]). The operation T is given by the product of matrices. It is not a commmutative group. Notice that $S O(3)$ as defined above is not a specific realization (although it could be perceived as such) of the group but its definition (see the subsection on realizations shortly hereafter). From a robotics and mechanics perspective the $S O(3)$ group describes the group of rotations of a rigid body with a fixed point in space. From the perspective of a manifold, it is possibly to locally describe an element of $S O(3)$ using three angles (the Euler angles for instance). Notice that there exists a two to one and onto homomorphism

$$
S U(2) \rightarrow S O(3),
$$

linking $S O(3)$ with the special unitary group $S U(2)$ (which is also a Lie group, i.e. a manifold and a group) which is isomorphic to the group of quaternions of unit norm [52].

Appendix A.3.2. SE(3) and Subgroups pf SE(3)

$\mathrm{SE}(3)$ is the proper Euclidean group of the space $\mathbb{R}^{3}$, or special Euclidean group, which is isomorphic to the semi-direct product of $\mathbb{R}^{3}$ and $S O(3)$, namely

$$
S E(3)=S O(3) \rtimes \mathbb{R}^{3},
$$

where the homomorphism $\tau$ takes an element $M \in S O(3)$ and maps it to the endomorphism

$$
t_{1} \times t_{2} \rightarrow M t_{1}+t_{2}
$$

Moreover, $T^{(1)}$ is the matrix product and $T^{(2)}$ is the addition of vectors of $\mathbb{R}^{3}$ so that $\mathrm{T}$ is defined on pairs $\left(M_{i}, t_{i}\right)$, $i=1,2, M_{i} \in S O(3)$ and $t_{i} \in \mathbb{R}^{3}$ and follows the aforementioned definition according to

$$
\left(M_{1}, t_{1}\right) \top\left(M_{2}, t_{2}\right)=\left(M_{1} M_{2}, M_{2} t_{1}+t_{2}\right) .
$$

By inspecting this last formula we can testify its usefulness since it describes the general motion of a rigid body, namely that any configuration can be achieved through the succession of a single rotation followed by a translation. Note that elements $(0, t)$ decribe pure translations and elements $(M, 0)$ rotations leaving a point fixed. The group formalism can be extended to more elaborate mechanisms and structures [53].

The subgroups are listed below together with some typical mechanism that move according to the subgroup (see [45], [53] for instance). The subgroup $H_{p}$ stand for the 1D helicoidal motion subgroup.

\begin{tabular}{c|c|c|c|c} 
Dim. & \multicolumn{3}{|c}{ Subgroups } \\
\hline 1 & $\mathbb{R}$ & $S O(2)$ & $H_{p}$ & \\
2 & $\mathbb{R}^{2}$ & $S O(2) \times \mathbb{R}$ & & \\
3 & $\mathbb{R}^{3}$ & $S O(2) \rtimes \mathbb{R}^{2}=S E(2)$ & $S O(3)$ & $H_{p} \rtimes \mathbb{R}^{2}$ \\
4 & $S O(2) \rtimes \mathbb{R}^{3}=S E(2) \times \mathbb{R}$ & & &
\end{tabular}




\begin{tabular}{c|c|c|c|c} 
Dim. & \multicolumn{3}{|c}{ Corresponding mechanical motion } \\
\hline 1 & prismatic joint & revolute joint & nut and bolt & \\
2 & planar transl. & cylindrical joint & & \\
3 & spatial transl. & planar rigid body & ball and socket & Y movement \\
4 & X movement & & &
\end{tabular}

\section{Appendix A.4. A note on realizations and Lie algebras}

It is sometimes useful to always use matrix algebra (for instance using the matrix product) rather than the specific operation $T$ which varies from case to case depending on the type of Lie group considered. In that respect, representation theory plays an important role ([45] Chapter 7 and [52]). The idea is to use more space, that is to map the Lie group into a larger group but one for which the $T$ product becomes the matrix multiplication. For instance in the case of $S E(3)$ it is possible to embed this group into the larger general linear group $G L(4)$ (the group of matrices $\mathbb{R}^{4 \times 4}$ without any orthogonality constraints on the columns or rows and without any condition on its determinant) so that $T$ transforms into the product of matrices of $G L(4)$. This is achieved by

$$
(M, t) \rightarrow\left(\begin{array}{cc}
M & t \\
0 & 1
\end{array}\right)
$$

Going back to $S O(3)$, we have seen that this group is directly given as a matrix representation, one for which the matrix seems to be of minimal dimension. It is nevertheless also possible to associate any element of $S O(3)$ with a subset of matrices of higher dimension namely $G L(6)$ which at times might be useful to represent this group as a subgroup of $S E(3)$ (see for instance [45]). In the process of going from the T product to the product of matrices it is sometimes more convenient to first represent the infinitesimal motions of the group (i.e. the Lie algebra $\mathfrak{s p}(3), \mathfrak{s e}(3)$ etc.) and then to see the corresponding representation of the groups (i.e. $S O(3), S E(3)$, etc.). 